\title{
Efficient Numerical Computation of Time-Fractional Nonlinear Schrödinger Equations in Unbounded Domain
}

\author{
Jiwei Zhang ${ }^{1}$, Dongfang $\mathrm{Li}^{2,3}$ and Xavier Antoine ${ }^{4, *}$ \\ ${ }^{1}$ Beijing Computational Science Research Center, Beijing 100193, P.R. China. \\ 2 School of Mathematics and Statistics, Huazhong University of Science and \\ Technology, Wuhan 430074, China. \\ ${ }^{3}$ Hubei Key Laboratory of Engineering Modeling and Scientific Computing, Huazhong \\ University of Science and Technology, Wuhan 430074, China. \\ ${ }^{4}$ Institut Elie Cartan de Lorraine, UMR CNRS 7502, Université de Lorraine, Inria \\ Nancy-Grand Est, SPHINX Team, F-54506 Vandoeuvre-lès-Nancy Cedex, France.
}

Received 12 September 2017; Accepted (in revised version) 18 December 2017

\begin{abstract}
The aim of this paper is to derive a stable and efficient scheme for solving the one-dimensional time-fractional nonlinear Schrödinger equation set in an unbounded domain. We first derive absorbing boundary conditions for the fractional system by using the unified approach introduced in $[47,48]$ and a linearization procedure. Then, the initial boundary-value problem for the fractional system with ABCs is discretized, a stability analysis is developed and the error estimate $\mathcal{O}\left(h^{2}+\tau\right)$ is stated. To accelerate the L1-scheme in time, a sum-of-exponentials approximation is introduced to speed-up the evaluation of the Caputo fractional derivative. The resulting algorithm is highly efficient for long time simulations. Finally, we end the paper by reporting some numerical simulations to validate the properties (accuracy and efficiency) of the derived scheme.
\end{abstract}

AMS subject classifications: 35R11, 35Q41, 65L20

Key words: Time-fractional nonlinear Schrödinger equation, absorbing boundary condition, stability analysis, convergence analysis, sum-of-exponentials approximation.

\section{Introduction}

The classical Schrödinger equation serves as the Feynman propagator for nonrelativistic quantum mechanics by using a Gaussian probability distribution in the space of all possible paths. This provides a useful mechanism that accounts naturally for the nonGaussian distributions corresponding to fractional structures. By extending the Feynman

\footnotetext{
*Corresponding author. Email addresses: jwzhang@csrc.ac.cn (J. Zhang), dfli@hust.edu.cn (D. Li),
} xavier.antoine@univ-lorraine.fr (X. Antoine) 
path integral from Gaussian distribution to Lévy-like quantum mechanical paths, Laskin [28, 29] proposed a space fractional Schrödinger equation. Naber [39] built the Time Fractional Schrödinger Equation (TFSE) in analogy with the fractional Fokker-Planck equation as well as with the application of the time Wich rotation. Related works have next been developed $[10,15,32,35,40,45]$, including e.g. the generalization of the TFSE to a full space and some new results on the correct continuity equation for the probability density. The fractional nonlinear Schrödinger equation is used to describe the nonlocal quantum phenomena in quantum physics and explore the quantum behaviors of either long-range interactions or multi-scale time-dependent processes. Many possible applications explain why this new direction in quantum physics is rapidly emerging $[26,28,29,35,39,40,46]$. Some analytical and approximate solutions have first been considered for the TFSE [23,41]. Nevertheless, the complexity of the mathematical structure of the TFSE with different potentials and nonlinearities leads to extremely difficult detailed analytical studies of its non standard properties, which are sometimes impossible to derive. Therefore, efficient and accurate numerical simulations $[3,6,11,17,19]$ are urgently needed to understand them.

In this paper, we develop and analyze some original accurate and efficient numerical methods for computing the solution to the general 1D Time-Fractional Nonlinear Schrödinger Equation (TFNSE)

$$
\begin{cases}\mathrm{i}_{0}^{C} \mathcal{D}_{t}^{\alpha} \psi(x, t)=-\psi_{x x}+V(x) \psi+f\left(|\psi|^{2}\right) \psi, & (x, t) \in \mathbb{R} \times(0, T], \\ \psi(x, 0)=\psi_{0}(x), & x \in \mathbb{R}, \\ \psi(x, t) \rightarrow 0 \text { when } \quad|x| \rightarrow+\infty, \quad t \in(0, T], & \end{cases}
$$

where $\mathrm{i}=\sqrt{-1}$ and $V(x)$ is the external potential. The function $f$ models general nonlinear effects with respect to $\psi$, e.g. for the case of the cubic nonlinearity $f\left(|\psi|^{2}\right) \psi=g|\psi|^{2} \psi$ that arises in nonlinear optics. If $g=+1$, one gets the well-known defocusing nonlinearity while $g=-1$ corresponds to the focusing situation. The operator ${ }_{0}^{C} \mathcal{D}_{t}^{\alpha}$ denotes the Caputo fractional derivative of order $\alpha(0<\alpha<1)$ with respect to $t[49]$ and given by

$$
{ }_{0}^{C} \mathcal{D}_{t}^{\alpha} \psi(x, t)=\frac{1}{\Gamma(1-\alpha)} \int_{0}^{t} \frac{1}{(t-s)^{\alpha}} \frac{\partial \psi(x, s)}{\partial s} d s, \quad 0<\alpha<1,
$$

where $\Gamma(\cdot)$ is the Gamma special function. Three crucial and fundamental difficulties appear when numerically solving problem (1.1)

(i) the spatial domain is unbounded,

(ii) the TFNSE is a nonlinear equation,

(iii) the (nonlocal) Caputo derivative a priori needs a huge storage and a high computational cost when discretized.

Over the past few decades, many contributions were made to overcome the numerical difficulties arising from solving PDEs in unbounded domains. For example, the method 
of Artificial/Absorbing Boundary Conditions (ABCs) is a powerful way to (exactly or approximately) reformulate the initial problem in the unbounded domain under the form of another problem appropriately set in a finite domain [2]. The basic idea is to build a suitable $A B C$ to eliminate the waves striking a fictitious boundary introduced to truncate the computational domain. In the literature, a lot of attention has been directed towards the construction of ABCs for time-fractional linear PDEs $[7,12,14,18]$ as well as Schrödingertype equations $[2,4,5]$. However, the question of designing accurate and stable ABCs for the TFNSE remains open, mainly due to the presence of the nonlinear term $f$. Indeed, the Laplace transform which is the main ingredient for building ABCs cannot be applied directly to the nonlinear case. To overcome this problem, the unified approach has been proposed in $[47,48]$ to construct accurate ABCs for the standard nonlinear Schrödinger equation $(\alpha=1)$.

The presence of a nonlocal convolution in the expression of the exact/approximate $\mathrm{ABC}$ leads to extremely difficult questions regarding both the development and numerical analysis of the truncated initial boundary-value problem (IBVP), and this even for the standard linear Schrödinger equation [44]. In practice, only the sub-optimal estimate is proved (see [44]). In addition, the brute force discretization of the Caputo derivative induces a huge storage and a high computational cost for simulating the TFNSE. For instances, the L1-approximation scheme $[27,30,34,44]$ requires the storage of all the past values of the unknown function, and $\mathcal{O}(n)$ flops at the $n$-th time step and each spatial grid point. Thus, the average storage is $\mathcal{O}(N J)$ and the total computational cost is $\mathcal{O}\left(N^{2} J\right)$, where $N$ and $J$ are the total numbers of time steps and spatial grid points, respectively. As a consequence, this is a severe limitation for the long time simulation of time-fractional PDEs, which would be even worst for higher-dimensional problems. The summation of exponentials (SOEs) to approximate the convolution kernel turns out to be successful to highly speed-up the evaluation of the Caputo derivative [20,38]. Indeed, the exponential kernel can be rewritten as a sequence of ODE systems or under the form of a standard recurrence relation. As proved in [20], the resulting algorithm has a nearly optimal complexity, i.e. $\mathcal{O}\left(J N \log ^{2} N\right)$ flops and $\mathcal{O}\left(J \log ^{2} N\right)$ storage for solving the TFNSE.

The goal of this paper is to develop some new stable and efficient schemes to simulate the TFNSE defined in an unbounded domain and to develop the numerical analysis of the resulting scheme. To this aim, we first extend the unified approach to derive some new nonlinear ABCs for the TFNSE arising in system (1.1), and present some original a priori energy estimates for the truncated bounded problem. We next derive a discretization scheme by using the L1-approximation of the Caputo derivative and build a linearized finite-difference scheme by linearizing the nonlinear term and implementing the ABCs. The resulting scheme avoids any extra computational cost compared with an implicit scheme. In addition, we introduce the fast algorithm developed in [20] to speed-up the evaluation of the time convolution arising in the Caputo derivative. Consequently, this significantly reduces both the memory storage and computational cost of the overall algorithm while keeping an almost similar accuracy compared with the direct method. For the theoretical analysis, the corresponding error estimates is proved to be $\mathcal{O}\left(h^{2}+\tau\right)(\mathrm{di}-$ 
rect scheme) and $\mathcal{O}\left(h^{2}+\tau+\varepsilon\right)$ (accelerated scheme) by using a nontrivial and original discrete fractional-type Gronwall-inequality [31]. The absolute error $\varepsilon$ is chosen during the approximation of the kernel by using the SOEs for the fast algorithm, and is far smaller than the mesh sizes $h$ and $\Delta t$. This parameter then does not affect the standard accuracy of the scheme.

The paper is organized as follows. In Section 2, we propose the construction of ABCs for the TFNSE based on the unified approach. In Section 3, we derive some a priori error estimates for the resulting truncated IBVP. Section 4 is devoted to the discretization scheme and its numerical analysis. Some numerical examples in Section 5 allows to confirm the efficiency of the scheme and validate the error estimates. In addition, we provide some examples to illustrate the behavior of the solution of the TFNSE. Finally, Section 6 concludes the paper.

\section{Construction of nonlinear ABCs for the TFNSE}

Let us introduce a computational bounded domain $\Omega^{\text {int }}:=\left\{x_{\ell}<x<x_{r}\right\}$, with left and right finite fictitious boundaries $\Gamma^{\ell}:=\left\{x_{\ell}\right\}$ and $\Gamma^{r}:=\left\{x_{r}\right\}$. The two points $x_{\ell}$ and $x_{r}$ are chosen such that the initial data $\psi_{0}(x)$ is compactly supported in $\Omega^{\text {int }}$. We define the left and right half-spaces by $\Omega^{\ell}:=\left\{-\infty<x<x_{\ell}\right\}$ and $\Omega^{r}:=\left\{x_{r}<x<+\infty\right\}$, respectively, and the exterior domain by $\Omega^{\mathrm{ext}}=\Omega^{\ell} \cup \Omega^{r}$. Let us apply the basic ideas of the unified approach proposed in $[47,48]$ to construct some ABCs for the TFNSE:

(1) Firstly, the TFNSE arising in (1.1) is rewritten in operator form in $\Omega^{\text {ext }}$ as

$$
\mathrm{i}_{0}^{C} \mathcal{D}_{t}^{\alpha} \psi(x, t)=\mathcal{L} \psi(x, t)+\mathcal{N} \psi(x, t), \quad x \in \Omega^{\mathrm{ext}},
$$

where the linear and nonlinear operators are respectively given by

$$
\mathcal{L} \psi=-\psi_{x x} \quad \text { and } \quad \mathcal{N} \psi=V \psi+f\left(|\psi|^{2}\right) \psi
$$

Based on the operator splitting method [13], for a small time step, the original equation is considered as taking first a linear effect and next a nonlinear effect.

(2) On $\Omega^{\text {ext }}$, we approximate the linear operator $\mathcal{L}$ by an approximate one-way operator $\mathcal{L}^{\text {app }}$ (by distinguishing between the right- and left-traveling waves) which can absorb the wave striking the artificial boundaries. The one-directional operators $\mathcal{L}^{\text {app }}$ are then used to replace the original linear operator $\mathcal{L}$.

(3) Taking the time step infinitesimal, we obtain an approximate one-directional equation by coupling the approximate operator $\mathcal{L}^{\text {app }}$ with the nonlinear operator $\mathcal{N}$

$$
\mathrm{i}_{0}^{\mathrm{C}} \mathcal{D}_{t}^{\alpha} \psi(x, t)=\mathcal{L}^{\mathrm{app}} \psi(x, t)+\mathcal{N} \psi(x, t) .
$$


Finally, applying the approximate equation (2.2) at the fictitious boundaries, one gets some ABCs. Therefore, the main point now is to construct the one-directional operators $\mathcal{L}^{\text {app. }}$.

To derive an operator $\mathcal{L}^{\text {app }}$ approximating $\mathcal{L}$, we first consider the following timefractional linear Schrödinger equation (TFLSE) in the exterior domain

$$
\begin{aligned}
& \mathrm{i}_{0}^{C} \mathcal{D}_{t}^{\alpha} \psi(x, t)=\mathcal{L} \psi(x, t)=-\psi_{x x}(x, t), \quad x \in \Omega^{\mathrm{ext}}, \\
& \psi_{0}(x)=0, \quad x \in \Omega^{\mathrm{ext}}, \\
& \psi(x, t) \rightarrow 0, \text { when } \quad|x| \rightarrow+\infty .
\end{aligned}
$$

Let us recall that the Laplace transform of the Caputo fractional derivative [49] is

$$
{ }_{0}^{C} \mathcal{D}_{t}^{\alpha[\psi(t)]}(s)=s^{\alpha} \widehat{\psi}(s)-s^{\alpha-1} \psi(0),
$$

where the Laplace transform is defined by

$$
\widehat{\psi}(s)=\int_{0}^{+\infty} \mathbf{e}^{-s t} \psi(t) d t, \quad \Re(s)>0 .
$$

Thus Eq. (2.3) can be written in the Laplace domain as

$$
\text { is }{ }^{\alpha} \widehat{\psi}(x, s)=-\widehat{\psi}_{x x}(x, s), \quad \Re(s)>0 .
$$

Setting $\sqrt{-\mathrm{i}}=\mathbf{e}^{-\frac{\pi \mathrm{i}}{4}}$, the general form of the solution to Eq. (2.7) is

$$
\widehat{\psi}(x, s)=A_{-}(s) \mathbf{e}^{-\frac{\pi \mathrm{i}}{4}} \mathbf{e}^{-\sqrt{s^{\alpha}} x}+A_{+}(s) \mathbf{e}^{-\frac{\pi \mathrm{i}}{4}} \mathbf{e}^{\sqrt{s^{\alpha}} x},
$$

where the coefficients $A_{ \pm}(s)$ are two arbitrary analytic functions. From (2.5), we deduce that

$$
\widehat{\psi}(x, s)= \begin{cases}A_{+}(s) \mathbf{e}^{-\frac{\pi \mathrm{i}}{4}} \mathbf{e}^{\sqrt{s^{\alpha}} x}, & x \in \Omega^{\ell}, \\ A_{-}(s) \mathbf{e}^{-\frac{\pi \mathrm{i}}{4}} \mathbf{e}^{-\sqrt{s^{\alpha}} x}, & x \in \Omega^{r} .\end{cases}
$$

Differentiating $\widehat{\psi}(x, s)$ with respect to $x$, one gets the two following relations

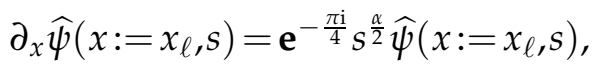

$$
\begin{aligned}
& \partial_{x} \widehat{\psi}\left(x:=x_{r}, s\right)=-\mathbf{e}^{-\frac{\pi \mathrm{i}}{4}} S^{\frac{\alpha}{2}} \widehat{\psi}\left(x:=x_{r}, s\right),
\end{aligned}
$$

which correspond to the Dirichlet-to-Neumann (DtN) maps at the interfaces for the TFLSE written in the Laplace domain.

Exact ABCs for the TFLSE. Applying the inverse Laplace transform to (2.9)-(2.10), one naturally gets the exact ABCs (DtN maps) for the TFLSE

$$
\partial_{\mathbf{n}} \psi(x, t)=-\frac{\mathbf{e}^{-\frac{\pi \mathrm{i}}{4}}}{\Gamma\left(1-\frac{\alpha}{2}\right)} \int_{0}^{t} \frac{\partial_{s} \psi(x, s)}{(t-s)^{\frac{\alpha}{2}}} d s=-\mathbf{e}^{-\frac{\pi \mathrm{i}}{4}}{ }_{0}^{C} \mathcal{D}_{t}^{\frac{\alpha}{2}} \psi(x, t), \quad \text { on } \Gamma^{\ell, r} .
$$


In the above relation, we introduced $\partial_{\mathbf{n}}$ as the outwardly directed unit normal vector to $\Gamma^{\ell, r}$.

Approximate ABCs for the TFLSE. The exact ABCs (2.11) cannot be directly combined with the nonlinear term $\mathcal{N} \psi$. To localize boundary conditions, we proceed as in the standard approach originally introduced by Engquist and Majda [16] for wave-like equations to obtain some local ABCs. To this aim, we first introduce the diagonal $(P, P)$-Padé approximation of the function $\sqrt{s^{\alpha}}$ used in $[4,5,8,9,21]$

$$
\sqrt{s^{\alpha}}=s_{0}^{\frac{\alpha}{2}}\left[1-\sum_{p=1}^{P} \frac{A_{p}\left(s_{0}^{\alpha}-s^{\alpha}\right)}{s_{0}^{\alpha}-\left(s_{0}^{\alpha}-s^{\alpha}\right) B_{p}}\right]+E_{P}\left(s^{\alpha}\right),
$$

where $s_{0}$ is the expansion point, and the coefficients $\left\{A_{p}\right\}_{1 \leq p \leq P}$ and $\left\{B_{p}\right\}_{1 \leq p \leq P}$ are given by

$$
A_{p}=\frac{2}{2 P+1} \sin ^{2}\left(\frac{p \pi}{2 P+1}\right), \quad B_{p}=\cos ^{2}\left(\frac{p \pi}{2 P+1}\right) .
$$

The error estimate of the Padé approximation is established in [37], for all $s^{\alpha}>0$,

$$
E_{P}\left(s^{\alpha}\right)=2 \sqrt{s^{\alpha}}\left(\frac{\gamma^{2 P+1}\left(s^{\alpha}\right)}{1+\gamma^{2 P+1}\left(s^{\alpha}\right)}\right) \text {, with } \gamma\left(s^{\alpha}\right)=\frac{\sqrt{s^{\alpha}}-1}{\sqrt{s^{\alpha}}+1} \text {. }
$$

Here, we only consider the simplest case, i.e. $P=1$, and obtain the third-order diagonal Padé approximation

$$
\sqrt{s^{\alpha}} \approx s_{0}^{\frac{\alpha}{2}} \frac{s_{0}^{\alpha}+3 s^{\alpha}}{3 s_{0}^{\alpha}+s^{\alpha}}
$$

Replacing $\sqrt{s^{\alpha}}$ in (2.9) and (2.10) by the approximation (2.14), one gets

$$
\left(3 s_{0}^{\alpha}+s^{\alpha}\right) \partial_{x} \widehat{\psi}(x, s) \pm \mathbf{e}^{-\frac{\pi \mathrm{i}}{4}} s_{0}^{\frac{\alpha}{2}}\left(s_{0}^{\alpha}+3 s^{\alpha}\right) \widehat{\psi}(x, s)=0,
$$

where the plus (minus, respectively) sign in \pm represents the right(left, respectively)hand side boundary condition. After some simple algebraic calculations with (2.15), we deduce the relation

$$
s^{\alpha} \widehat{\psi}(x, s)=-\left(\partial_{x} \pm 3 \mathbf{e}^{-\frac{\pi \mathrm{i}}{4}} s_{0}^{\frac{\alpha}{2}}\right)^{-1}\left(3 s_{0}^{\alpha} \partial_{x} \pm \mathbf{e}^{-\frac{\pi \mathrm{i}}{4}} s_{0}^{\frac{\alpha}{2}} s_{0}^{\alpha}\right) \widehat{\psi}(x, s) .
$$

Applying the inverse Laplace transform to (2.16) and multiplying by i, one gets

$$
\mathrm{i}_{0}^{C} \mathcal{D}_{t}^{\alpha} \psi(x, t)=-\mathrm{i}\left(\partial_{x} \pm 3 \mathbf{e}^{-\frac{\pi \mathrm{i}}{4}} s_{0}^{\frac{\alpha}{2}}\right)^{-1}\left(3 s_{0}^{\alpha} \partial_{x} \pm \mathbf{e}^{-\frac{\pi \mathrm{i}}{4}} s_{0}^{\frac{\alpha}{2}} s_{0}^{\alpha}\right) \psi(x, t) .
$$

Comparing (2.17) with (2.3), we can derive the approximate operator

$$
\mathcal{L}^{\mathrm{app}}=-\mathrm{i}\left(\partial_{x} \pm 3 \mathbf{e}^{-\frac{\pi \mathrm{i}}{4}} s_{0}^{\frac{\alpha}{2}}\right)^{-1}\left(3 s_{0}^{\alpha} \partial_{x} \pm \mathbf{e}^{-\frac{\pi \mathrm{i}}{4}} S_{0}^{\frac{\alpha}{2}} s_{0}^{\alpha}\right) .
$$


Nonlinear approximate ABCs for the TFNSE. Substituting (2.18) into (2.2), the nonlinear ABCs for the TFNSE are

$$
\left(\partial_{x} \pm 3 \mathbf{e}^{-\frac{\pi \mathrm{i}}{4}} s_{0}^{\frac{\alpha}{2}}\right)_{0}^{C} \mathcal{D}_{t}^{\alpha} \psi+\left(3 s_{0}^{\alpha} \partial_{x} \pm \mathbf{e}^{-\frac{\pi \mathrm{i}}{4}} s_{0}^{\frac{\alpha}{2}} s_{0}^{\alpha}\right) \psi=-\mathrm{i}\left(\partial_{x} \pm 3 \mathbf{e}^{-\frac{\pi \mathrm{i}}{4}} s_{0}^{\frac{\alpha}{2}}\right)\left(V \psi+f\left(|\psi|^{2}\right) \psi\right) .
$$

By using an approach similar to the one by Kuska [25], we linearize the nonlinear term $f\left(|\psi|^{2}\right)$ to obtain a variety of boundary conditions:

$$
\left(\partial_{x} \pm 3 \mathbf{e}^{-\frac{\pi \mathrm{i}}{4}} S_{0}^{\frac{\alpha}{2}}\right)_{0}^{C} \mathcal{D}_{t}^{\alpha} \psi+\left(3 s_{0}^{\alpha} \partial_{x} \pm \mathbf{e}^{-\frac{\pi \mathrm{i}}{4}} S_{0}^{\frac{\alpha}{2}} s_{0}^{\alpha}\right) \psi=-\mathrm{i}\left(V+f\left(|\psi|^{2}\right)\right)\left(\partial_{x} \psi \pm 3 \mathbf{e}^{-\frac{\pi \mathrm{i}}{4}} s_{0}^{\frac{\alpha}{2}} \psi\right) .
$$

Finally, we obtain an IBVP in $\Omega^{\text {int }} \times[0, T]$ with the boundary conditions $(2.20)$ on $\Gamma^{\ell, r}$

$$
\begin{aligned}
& \mathrm{i}_{0}^{C} \mathcal{D}_{t}^{\alpha} \psi(x, t)=-\psi_{x x}+V \psi+f\left(|\psi|^{2}\right) \psi, \quad t>0, \quad x \in \Omega^{\mathrm{int}}, \\
& \psi(x, 0)=\psi_{0}(x), \quad x \in \Omega^{\mathrm{int}}, \\
& \left(\partial_{x} \mp 3 \mathbf{e}^{-\frac{\pi \mathrm{i}}{4}} s_{0}^{\frac{\alpha}{2}}\right)_{0}^{C} \mathcal{D}_{t}^{\alpha} \psi+\left(3 s_{0}^{\alpha} \partial_{x} \mp \mathbf{e}^{-\frac{\pi \mathrm{i}}{4}} s_{0}^{\frac{3 \alpha}{2}}\right) \psi=-\mathrm{i}\left(V+f\left(|\psi|^{2}\right)\right)\left(\partial_{x} \mp 3 \mathbf{e}^{-\frac{\pi \mathrm{i}}{4}} s_{0}^{\frac{\alpha}{2}}\right) \psi, \quad x \in \Gamma^{\ell, r} .
\end{aligned}
$$

Remark 2.1. Generally, the ABCs (2.19) perform better than (2.20). However, the stability analysis with (2.20) is easier to handle than with (2.19). The comparison is discussed in [50] for general nonlinear Schrödinger equations. In this paper, we consider (2.20) as $\mathrm{ABC}$.

\section{A priori error estimates}

To state the stability of (2.21)-(2.23), we introduce two auxiliary variables

$$
\begin{aligned}
& \theta(t)=\partial_{x} \psi\left(x_{\ell}, t\right)-3 s_{0}^{\frac{\alpha}{2}} \mathbf{e}^{-\frac{\pi \mathrm{i}}{4}} \psi\left(x_{\ell}, t\right), \\
& \phi(t)=\partial_{x} \psi\left(x_{r}, t\right)+3 s_{0}^{\frac{\alpha}{2}} \mathbf{e}^{-\frac{\pi \mathrm{i}}{4}} \psi\left(x_{r}, t\right) .
\end{aligned}
$$

Thus, the reduced problem (2.21)-(2.23) can be equivalently rewritten as

$$
\begin{array}{lr}
\mathrm{i}_{0}^{C} \mathcal{D}_{t}^{\alpha} \psi(x, t)=-\psi_{x x}+V \psi+f\left(|\psi|^{2}\right) \psi, & x \in \Omega^{\mathrm{int}}, \quad t>0, \\
\psi(x, 0)=\psi_{0}(x), & x \in \Omega^{\mathrm{int},}, \\
\psi_{x}(x, t)=\theta(t)+3 s_{0}^{\frac{\alpha}{2}} \mathbf{e}^{-\frac{\pi \mathrm{i}}{4}} \psi(x, t), & x \in \Gamma^{\ell}, \\
{ }_{0}^{C} \mathcal{D}_{t}^{\alpha} \theta(t)+\mathrm{i}\left(V(x)+f\left(|\psi|^{2}\right)\right) \theta(t)+3 s_{0}^{\alpha} \theta(t)+8 s_{0}^{\frac{3 \alpha}{2}} \mathbf{e}^{-\frac{\pi \mathrm{i}}{4}} \psi(x, t)=0, & x \in \Gamma^{\ell}, \\
\psi_{x}(x, t)=\phi(t)-3 s_{0}^{\frac{\alpha}{2}} \mathbf{e}^{-\frac{\pi \mathrm{i}}{4}} \psi(x, t), & x \in \Gamma^{r}, \\
{ }_{0}^{C} \mathcal{D}_{t}^{\alpha} \phi(t)+\mathrm{i}\left(V+f\left(|\psi|^{2}\right)\right) \phi(t)+3 s_{0}^{\alpha} \phi(t)-8 s_{0}^{\frac{3 \alpha}{2}} \mathbf{e}^{-\frac{\pi \mathrm{i}}{4}} \psi(x, t)=0, & x \in \Gamma^{r} .
\end{array}
$$

Some useful lemmas are now given to state a priori estimate for the reduced problem (3.1)-(3.6). 
Lemma 3.1 ([33]). Let $u(t)$ be a complex-valued function which is absolutely continuous on $[0, T]$. Then, the following inequality holds

$$
{ }_{0}^{C} \mathcal{D}_{t}^{\alpha}|u(t)|^{2} \leq \bar{u}(t){ }_{0}^{C} \mathcal{D}_{t}^{\alpha} u(t)+u(t){ }_{0}^{C} \mathcal{D}_{t}^{\alpha} \bar{u}(t), \quad 0<\alpha<1,
$$

where $|u|^{2}=\bar{u} u$ and $\bar{u}$ represents the complex conjugate function of $u$.

Now, let us consider the following lemma (see e.g. [42]).

Lemma 3.2. Let $0<\alpha<1$. We assume that $y(t)$ is a nonnegative absolutely continuous function that satisfies

$$
{ }_{0}^{C} \mathcal{D}_{t}^{\alpha} y(t) \leq c_{1} y(t)+c_{2}(t), \quad 0<\alpha<1, \quad t \in(0, T],
$$

where $c_{1}>0$ and $c_{2}(t)$ is an integrable nonnegative function on $[0, T]$. Then, we have

$$
y(t) \leq y(0) E_{\alpha, 1}\left(c_{1} t^{\alpha}\right)+\Gamma(\alpha) E_{\alpha, \alpha}\left(c_{1} t^{\alpha}\right)_{0} \mathcal{D}_{t}^{-\alpha} c_{2}(t),
$$

where $E_{\alpha, \beta}(z)=\sum_{n=0}^{\infty} \frac{z^{n}}{\Gamma(n \alpha+\beta)}$ are the Mittag-Leffler functions and $\mathcal{D}_{t}^{-\alpha} u(t)$ is the RiemannLiouville integral given by

$$
{ }_{0} \mathcal{D}_{t}^{-\alpha} u(t)=\frac{1}{\Gamma(\alpha)} \int_{0}^{t} \frac{u(\tau)}{(t-\tau)^{1-\alpha}} d \tau, \quad 0<\alpha<1 .
$$

We can then deduce the following result.

Theorem 3.1. Let $(\psi(x, t), \phi(t), \theta(t))$ be the solution of the reduced problem (3.1)-(3.6). Then, for $0<\alpha<1$, we have the following a priori estimate

$$
\int_{x_{\ell}}^{x_{r}}|\psi(x, t)|^{2} d x+\frac{1}{8} s_{0}^{-\frac{3 \alpha}{2}}\left(|\phi(t)|^{2}+|\theta(t)|^{2}\right) \leq \int_{x_{\ell}}^{x_{r}}\left|\psi_{0}(x)\right|^{2} d x .
$$

Proof. By multiplying (3.1) by $\bar{\psi}(x, t)$, integrating by parts the result over $\Omega^{\text {int }}$, taking the complex conjugate of (3.1), multiplying the result by $\psi(x, t)$ and integrating by parts over $\Omega^{\text {int }}$, and finally combining the two equations and taking the complex parts, we have

$$
\int_{x_{\ell}}^{x_{r}}\left[\bar{\psi}(x, t)_{0}^{C} \mathcal{D}_{t}^{\alpha} \psi(x, t)+\psi(x, t)_{0}^{C} \mathcal{D}_{t}^{\alpha} \bar{\psi}(x, t)\right] d x=-\left.2 \operatorname{Im}\left\{\bar{\psi} \psi_{x}\right\}\right|_{x_{\ell}} ^{x_{r}}
$$

Substituting $\psi_{x}\left(x_{\ell}, t\right)$ and $\psi_{x}\left(x_{r}, t\right)$, defined by (3.3) and (3.5), into (3.11), we arrive at

$$
\begin{aligned}
& \int_{x_{\ell}}^{x_{r}}\left[\bar{\psi}(x, t)_{0}^{C} \mathcal{D}_{t}^{\alpha} \psi(x, t)+\psi(x, t)_{0}^{C} \mathcal{D}_{t}^{\alpha} \bar{\psi}(x, t)\right] d x \\
= & -3 \sqrt{2} s_{0}^{\frac{\alpha}{2}}\left(\left|\psi\left(x_{\ell}, t\right)\right|^{2}+\left|\psi\left(x_{r}, t\right)\right|^{2}\right)+2 \operatorname{Im}\left\{\mathbf{e}^{-\frac{\pi \mathrm{i}}{4}}\left(\phi(t) \bar{\psi}\left(x_{r}, t\right)-\theta \bar{\psi}\left(x_{\ell}, t\right)\right)\right\} .
\end{aligned}
$$


Multiplying (3.6) by $\frac{1}{8} s_{0}^{-\frac{3 \alpha}{2}} \bar{\phi}\left(x_{r}, t\right)$, then taking the complex conjugate of (3.6), multiplying the result by $\frac{1}{8} s_{0}^{-\frac{3 \alpha}{2}} \phi\left(x_{r}, t\right)$, combining the two equations, taking the real parts, and using the same arguments as (3.4), we obtain

$$
\begin{aligned}
& \frac{1}{8} s_{0}^{-\frac{3 \alpha}{2}}\left[\bar{\theta}(t)_{0}^{C} \mathcal{D}_{t}^{\alpha} \theta(t)+\theta(t)_{0}^{C} \mathcal{D}_{t}^{\alpha} \bar{\theta}(t)\right]=-\frac{3}{4} s_{0}^{-\frac{\alpha}{2}}|\theta(t)|^{2}-2 \operatorname{Re}\left\{\mathbf{e}^{-\frac{\pi \mathrm{i}}{4}} \theta(t) \bar{\psi}\left(x_{\ell}, t\right)\right\} \\
& \frac{1}{8} s_{0}^{-\frac{3 \alpha}{2}}\left[\bar{\phi}(t)_{0}^{C} \mathcal{D}_{t}^{\alpha} \phi(t)+\phi(t)_{0}^{C} \mathcal{D}_{t}^{\alpha} \bar{\phi}(t)\right]=-\frac{3}{4} s_{0}^{-\frac{\alpha}{2}}|\phi(t)|^{2}+2 \operatorname{Re}\left\{\mathbf{e}^{-\frac{\pi \mathrm{i}}{4}} \phi(t) \bar{\psi}\left(x_{r}, t\right)\right\} .
\end{aligned}
$$

Adding (3.12), (3.13) and (3.14), applying Lemma 3.1 and noticing that $|\operatorname{Im}\{Z\}|+|\operatorname{Re}\{Z\}| \leq$ $\sqrt{2}|Z|$ lead to

$$
\begin{aligned}
& { }_{0}^{C} \mathcal{D}_{t}^{\alpha} \int_{x_{\ell}}^{x_{r}}|\psi(x, t)|^{2} d x+\frac{1}{8} s_{0}{ }^{-\frac{3 \alpha}{2}}{ }_{0}^{C} \mathcal{D}_{t}^{\alpha}\left[|\phi(t)|^{2}+|\theta(t)|^{2}\right] \\
\leq & -3 \sqrt{2} s_{0}^{\frac{\alpha}{2}}\left(\left|\psi\left(x_{\ell}, t\right)\right|^{2}+\left|\psi\left(x_{r}, t\right)\right|^{2}\right)-\frac{3}{4} s_{0}^{-\frac{\alpha}{2}}\left(|\theta(t)|^{2}+|\phi(t)|^{2}\right)+2 \sqrt{2}(|\phi(t) \bar{\psi}|+|\theta \bar{\psi}|) .
\end{aligned}
$$

Applying the Cauchy-Schwarz inequality

$$
\left|\theta(t) \bar{\psi}\left(x_{\ell}, t\right)\right| \leq \frac{1}{4 \epsilon}|\theta(t)|^{2}+\epsilon\left|\psi\left(x_{\ell}, t\right)\right|^{2} \quad \text { and } \quad\left|\phi(t) \bar{\psi}\left(x_{r}, t\right)\right| \leq \frac{1}{4 \epsilon}|\phi(t)|^{2}+\epsilon\left|\psi\left(x_{r}, t\right)\right|^{2},
$$

with $\epsilon=\frac{3}{2} s_{0}^{\frac{\alpha}{2}}$ to Eq. (3.15), we get

$$
{ }_{0}^{C} \mathcal{D}_{t}^{\alpha}\left[\int_{x_{\ell}}^{x_{r}}|\psi(x, t)|^{2} d x+\frac{1}{8} s_{0}^{-\frac{3 \alpha}{2}}\left(|\phi(t)|^{2}+|\theta(t)|^{2}\right)\right] \leq-\frac{9}{24} s_{0}^{-\frac{\alpha}{2}}\left(|\phi(t)|^{2}+|\theta(t)|^{2}\right) \leq 0 .
$$

Finally applying Lemma 3.2 completes the proof.

\section{Discretization and analysis of the schemes}

The section focuses on the construction and numerical analysis of the linearized numerical schemes. Let $\tau=T / N$ and $h=\left(x_{\ell}-x_{r}\right) / J$ be the temporal and spatial step sizes, respectively, where $N$ and $J$ are two given positive integers. We denote the discrete time by $t_{n}=n \tau(0 \leq n \leq N)$ and $\Omega_{\tau}=\left\{t_{n} \mid 0=t_{0}<t_{1}<\cdots<t_{N}=T\right\}$. A point of the uniform spatial grid is such that $x_{j}=j h,(0 \leq j \leq J)$, and $\Omega_{h}=\left\{x_{j} \mid x_{j}=x_{\ell}+j h, 0 \leq j \leq J\right\}$. We also set

$$
\psi_{j}^{n}=\psi\left(x_{j}, t_{n}\right), \quad V_{j}=V\left(x_{j}\right), \quad \phi^{n}=\phi\left(t_{n}\right), \quad \theta^{n}=\theta\left(t_{n}\right) .
$$

\subsection{Derivation of the linearized L1-scheme}

The L1-scheme for approximating the Caputo fractional derivative is

$$
{ }_{0}^{C} \mathcal{D}_{t_{n}}^{\alpha} v=\frac{1}{\Gamma(1-\alpha)} \int_{0}^{t_{n}} \frac{v^{\prime}(s)}{\left(t_{n}-s\right)^{\alpha}} d s=\frac{\tau^{-\alpha}}{\Gamma(2-\alpha)} \sum_{k=1}^{n} a_{n-k}\left(v^{k}-v^{k-1}\right)+Q^{n},
$$


where $v^{n}=v\left(t_{n}\right)$ and $a_{k}=(k+1)^{1-\alpha}-k^{1-\alpha}, k \geq 0$.

Denote the L1 scheme by

$$
{ }_{0}^{C} \mathbb{D}_{\tau}^{\alpha}=\frac{\tau^{-\alpha}}{\Gamma(2-\alpha)} \sum_{k=1}^{n} a_{n-k}\left(v^{k}-v^{k-1}\right) .
$$

If $v \in C^{2}([0, T])$, the truncation error $Q^{n}$ satisfies $[34,44]$

$$
\left|Q^{n}\right| \leq C \tau^{2-\alpha} \text {. }
$$

For a sequence of functions $\left\{w_{j}^{n}\right\}_{0 \leq n \leq N, 0 \leq j \leq J}$, we define

$$
\begin{aligned}
& \delta_{x} w_{j+\frac{1}{2}}^{n}=\frac{w_{j+1}^{n}-w_{j}^{n}}{h}, \quad \delta_{x}^{2} w_{j}^{n}=\frac{1}{h}\left(\delta_{x} w_{j+\frac{1}{2}}-\delta_{x} w_{j-\frac{1}{2}}\right), \\
& \delta_{t} w_{j}^{n}=\frac{w_{j}^{n}-w_{j}^{n-1}}{\tau}, \quad{ }_{0}^{C} \mathbb{D}_{\tau}^{\alpha} w_{j}^{n}:=\frac{\tau^{-\alpha}}{\Gamma(2-\alpha)} \sum_{k=1}^{n} a_{n-k}\left(w_{j}^{k}-w_{j}^{k-1}\right) .
\end{aligned}
$$

Writing the TFNSE equation at the left point $x_{\ell}$

$$
i_{0}^{C} \mathcal{D}_{t}^{\alpha} \psi_{0}^{n}=-\left(\psi_{0}^{n}\right)_{x x}+V_{0} \psi_{0}^{n}+f\left(\left|\psi_{0}^{n}\right|^{2}\right) \psi_{0}^{n},
$$

and using the Taylor expansion, we have

$$
\begin{aligned}
\left(\psi_{0}^{n}\right)_{x} & =\frac{1}{h}\left(\psi_{1}^{n}-\psi_{0}^{n}\right)-\frac{h}{2}\left(\psi_{0}^{n}\right)_{x x}+\mathcal{O}\left(h^{2}\right) \\
& =\delta_{x} \psi_{\frac{1}{2}}^{n}+\frac{h}{2}\left(i_{0}^{C} \mathcal{D}_{t}^{\alpha} \psi_{0}^{n}-\left(V_{0}+f\left(\left|\psi_{0}^{n}\right|^{2}\right)\right) \psi_{0}^{n}\right)+\mathcal{O}\left(h^{2}\right) .
\end{aligned}
$$

Similarly, at the right boundary point $x_{J}$, we have

$$
\left(\psi_{J}^{n}\right)_{x}=\delta_{x} \psi_{J-\frac{1}{2}}^{N}-\frac{h}{2}\left(i_{0}^{C} \mathcal{D}_{t}^{\alpha} \psi_{J}^{n}-\left(V_{J}+f\left(\left|\psi_{J}^{n}\right|^{2}\right)\right) \psi_{J}^{n}\right)+\mathcal{O}\left(h^{2}\right) .
$$

We apply the L1-scheme to approximate the time-fractional Caputo derivative, the second-order central finite difference method to approximate the second-order spatial derivative at the interior points, and use (4.3) and (4.4) to couple the ABCs and the linearized approach to deal with the nonlinear term. This yields

$$
\begin{aligned}
& \mathrm{i}{ }_{0}^{C} \mathbb{D}_{\tau}^{\alpha} \psi_{j}^{n}=-\delta_{x}^{2} \psi_{j}^{n}+\left[V_{j}+f\left(\left|\psi_{j}^{n-1}\right|^{2}\right)\right] \psi_{j}^{n}+T_{j}^{n}, \quad 1 \leq j \leq J-1, \quad 1 \leq n \leq N, \\
& \mathrm{i} \frac{h}{2}{ }_{0}^{C} \mathbb{D}_{\tau}^{\alpha} \psi_{0}^{n}=-\delta_{x} \psi_{\frac{1}{2}}^{n}+\left(\theta^{n}+3 s_{0}^{\frac{\alpha}{2}} \mathbf{e}^{-\frac{\pi \mathrm{i}}{4}} \psi_{0}^{n}\right)+\frac{h}{2}\left[V_{0}+f\left(\left|\psi_{0}^{n-1}\right|^{2}\right)\right] \psi_{0}^{n}+T_{0}^{n}, \\
& { }_{0}^{C} \mathbb{D}_{\tau}^{\alpha} \theta^{n}=-\mathrm{i}\left[V_{0}+f\left(\left|\psi_{0}^{n-1}\right|^{2}\right)\right] \theta^{n}-3 s_{0}^{\alpha} \theta^{n}-8 s_{0}^{\frac{3 \alpha}{2}} \mathbf{e}^{-\frac{\pi \mathrm{i}}{4}} \psi_{0}^{n}+R_{0}^{n}, \\
& \mathrm{i} \frac{h}{2}{ }_{0}^{C} \mathbb{D}_{\tau}^{\alpha} \psi_{J}^{n}=\delta_{x} \psi_{J-\frac{1}{2}}^{n}+\left(3 s_{0}^{\frac{\alpha}{2}} \mathbf{e}^{-\frac{\pi \mathrm{i}}{4}} \psi_{J}^{n}-\phi^{n}\right)+\frac{h}{2}\left[V_{J}+f\left(\left|\psi_{J}^{n-1}\right|^{2}\right)\right] \psi_{J}^{n}+T_{J}^{n}, \\
& { }_{0}^{C} \mathbb{D}_{\tau}^{\alpha} \phi^{n}=-\mathrm{i}\left[V_{J}+f\left(\left|\psi_{J}^{n-1}\right|^{2}\right)\right] \phi^{n}-3 s_{0}^{\alpha} \phi^{n}+8 s_{0}^{\frac{3 \alpha}{2}} \mathbf{e}^{-\frac{\pi \mathrm{i}}{4}} \psi_{J}^{n}+R_{J}^{n}, \\
& \psi_{j}^{0}=\psi_{0}\left(x_{j}\right), \quad 0 \leq j \leq J .
\end{aligned}
$$


Applying the Taylor expansion and (4.1), we have

$$
\begin{aligned}
& \left|T_{j}^{n}\right| \leq C\left(\tau+h^{2}\right), \quad 1 \leq j \leq J-1, \quad 1 \leq n \leq N \\
& \left|T_{0}^{n}\right| \leq C\left(\tau+h^{2}\right), \quad\left|T_{J}^{n}\right| \leq C\left(\tau+h^{2}\right), \quad 1 \leq n \leq N, \\
& \left|R_{0}^{n}\right| \leq C \tau, \quad\left|R_{J}^{n}\right| \leq C \tau, \quad 1 \leq n \leq N .
\end{aligned}
$$

Omitting the small terms in (4.5)-(4.10) based on the above error bounds, we construct the following finite difference scheme for solving problem (3.1)-(3.4) by

$$
\begin{aligned}
& \mathrm{i}{ }_{0}^{C} \mathbb{D}_{\tau}^{\alpha} \Psi_{j}^{n}=-\delta_{x}^{2} \Psi_{j}^{n}+\left[V_{j}+f\left(\left|\Psi_{j}^{n-1}\right|^{2}\right)\right] \Psi_{j}^{n}, \quad 1 \leq j \leq J-1, \quad 1 \leq n \leq N, \\
& \mathrm{i} \frac{h}{2}{ }_{0}^{C} \mathbb{D}_{\tau}^{\alpha} \Psi_{0}^{n}=-\delta_{x} \Psi_{\frac{1}{2}}^{n}+\left(\Theta^{n}+3 s_{0}^{\frac{\alpha}{2}} \mathbf{e}^{-\frac{\pi \mathrm{i}}{4}} \Psi_{0}^{n}\right)+\frac{h}{2}\left[V_{0}+f\left(\left|\Psi_{0}^{n-1}\right|^{2}\right)\right] \Psi_{0}^{n}, \\
& { }_{0}^{C} \mathbb{D}_{\tau}^{\alpha} \Theta^{n}=-\mathrm{i}\left[V_{0}+f\left(\left|\Psi_{0}^{n-1}\right|^{2}\right)\right] \Theta^{n}-3 s_{0}^{\alpha} \Theta^{n}-8 s_{0}^{\frac{3 \alpha}{2}} \mathbf{e}^{-\frac{\pi \mathrm{i}}{4}} \Psi_{0}^{n}, \\
& \mathrm{i} \frac{h}{2}{ }_{0}^{C} \mathbb{D}_{\tau}^{\alpha} \Psi_{J}^{n}=\delta_{x} \Psi_{J-\frac{1}{2}}^{n}+\left(3 s_{0}^{\frac{\alpha}{2}} \mathbf{e}^{-\frac{\pi \mathrm{i}}{4}} \Psi_{J}^{n}-\Phi^{n}\right)+\frac{h}{2}\left[V_{J}+f\left(\left|\Psi_{J}^{n-1}\right|^{2}\right)\right] \Psi_{J}^{n}, \\
& { }_{0}^{C} \mathbb{D}_{\tau}^{\alpha} \Phi^{n}=-\mathrm{i}\left[V_{J}+f\left(\left|\Psi_{J}^{n-1}\right|^{2}\right)\right] \Phi^{n}-3 s_{0}^{\alpha} \Phi^{n}+8 s_{0}^{\frac{3 \alpha}{2}} \mathbf{e}^{-\frac{\pi \mathrm{i}}{4}} \Psi_{J}^{n}, \\
& \Psi_{j}^{0}=\Psi_{0}\left(x_{j}\right), \quad 0 \leq j \leq J,
\end{aligned}
$$

where $\Psi_{j}^{n}, \Phi^{n}$ and $\Theta^{n}$ correspond to the numerical approximations of $\psi_{j}^{n}, \phi^{n}$ and $\theta^{n}$, respectively.

\subsection{Numerical analysis of the linearized L1-scheme}

Let us now consider the stability and convergence analysis of the finite difference scheme. Let $u=\left\{\left(u_{0}, u_{1}, \cdots, u_{J}\right)\right\}$ and $v=\left\{\left(v_{0}, v_{1}, \cdots, v_{J}\right)\right\}$ defined on $\Omega_{h}$. We introduce the following inner products and norms

$$
\begin{aligned}
& (u, v)=h\left(\frac{1}{2} \bar{u}_{0} v_{0}+\sum_{j=1}^{J-1} \bar{u}_{j} v_{j}+\frac{1}{2} \bar{u}_{j} v_{J}\right), \quad\|u\|=\sqrt{(u, u)}, \\
& |u|_{1}=\sqrt{h \sum_{j=1}^{J}\left|\delta_{x} \bar{u}_{j-\frac{1}{2}}\right|\left|\delta_{x} u_{j-\frac{1}{2}}\right|, \quad\|u\|_{\infty}=\max _{0 \leq j \leq J}\left|u_{j}\right| .}
\end{aligned}
$$

Lemma 4.1 ([24]). For any discrete function $v \in \Omega_{h}$, the following inequality holds

$$
\|v\|_{\infty}^{2} \leq \epsilon|v|_{1}^{2}+\left(\frac{1}{\epsilon}+\frac{1}{x_{r}-x_{\ell}}\right)\|v\|^{2} \quad \text { for any } \epsilon>0
$$

Lemma 4.2 ([31]). Suppose that $\left\{\omega^{n}\right\}_{n=0, \ldots}$ and $\left\{g^{n}\right\}_{n=0, \ldots}$ are two nonnegative sequences that satisfy $\omega^{0} \leq g^{0}$ and

$$
{ }_{0}^{C} \mathbb{D}_{\tau}^{\alpha} \omega^{n} \leq \lambda_{1} \omega^{n}+\lambda_{2} \omega^{n-1}+g^{n}, \quad n \geq 1,
$$


where $\lambda_{1}>0$ and $\lambda_{2} \geq 0$ are two given real-valued constants. Then, there exists a constant $\tau^{*}$ such that, for $\tau \leq \tau^{*}$,

$$
\omega^{n} \leq 2\left(\omega^{0}+\frac{t_{n}^{\alpha}}{\Gamma(1+\alpha)} \max _{0 \leq j \leq n} g^{j}\right) E_{\alpha}\left(2 \lambda t_{n}\right), \quad 1 \leq n \leq N,
$$

where $E_{\alpha}(z)=E_{\alpha, 1}(z)$ and $\lambda=\lambda_{1}+\frac{\lambda_{2}}{\lambda_{1}\left(a_{0}-a_{1}\right)}$.

Lemma 4.3. Let $\left\{v^{n}\right\}_{n=0}^{N}$ be a sequence of functions defined in $\Omega_{\tau}$. If $v_{0} \leq \kappa$ and

$$
\operatorname{Re}\left({ }_{0}^{C} \mathbb{D}_{\tau}^{\alpha} v^{n}, v^{n}\right) \leq C\left\|v^{n-1}\right\|^{2}+C\left\|v^{n}\right\|^{2}+\kappa^{2}, \quad 2 \leq n \leq N,
$$

where $\kappa>0$ and $C$ is a positive constant bounded independently of $\tau$, then, there exists a positive constant $\tau_{*}$ such that, for $\tau<\tau_{*}$,

$$
\left\|v^{n}\right\| \leq C \kappa
$$

Proof. Using the definition of ${ }_{0}^{C} \mathbb{D}_{\tau}^{\alpha}$ and the facts that $a_{0}>a_{1}>\cdots>a_{n} \rightarrow 0$, we have

$$
\begin{aligned}
\operatorname{Re}\left({ }_{0}^{C} \mathbb{D}_{\tau}^{\alpha} v^{n}, v^{n}\right) & =\operatorname{Re}\left(\frac{\tau^{2-\alpha}}{\Gamma(2-\alpha)}\left(a_{0} v^{n}-\sum_{j=1}^{n-1}\left(a_{n-j-1}-a_{n-j}\right) v^{j}-a_{n-1} v^{0}, v^{n}\right)\right. \\
& \geq \frac{\tau^{2-\alpha}}{\Gamma(2-\alpha)}\left(a_{0}\left\|v^{n}\right\|^{2}-\sum_{j=1}^{n-1}\left(a_{n-j-1}-a_{n-j}\right) \frac{\left\|v^{j}\right\|^{2}+\left\|v^{n}\right\|^{2}}{2}-a_{n-1} \frac{\left\|v^{0}\right\|^{2}+\left\|v^{n}\right\|^{2}}{2}\right) \\
& =\frac{1}{2}{ }_{0}^{C} \mathbb{D}_{\tau}^{\alpha}\left\|v^{n}\right\|^{2} .
\end{aligned}
$$

Substituting (4.23) into (4.21) and using Lemma 4.2, we can easily get (4.22).

We now define the error function on the grid, for $0 \leq j \leq J, 0 \leq n \leq N$,

$$
e_{j}^{n}=\psi_{j}^{n}-\Psi_{j}^{n}, \quad \varphi^{n}=\phi^{n}-\Phi^{n}, \quad \vartheta=\theta^{n}-\Theta^{n} .
$$

Let $K=\max _{1 \leq n \leq N}\left\{\left\|\psi^{n}\right\|_{\infty}+\left|\phi^{n}\right|+\left|\theta^{n}\right|\right\}+1$. We have the following result.

Theorem 4.1. Let us assume that system (3.1)-(3.4) has unique smooth solutions $\psi(x, t), \theta(t)$ and $\phi(t)$ in $\Omega^{i n t} \times[0, T]$. Then, there exist two positive constants $\tau_{0}$ and $h_{0}$ such that, when $\tau \leq \tau_{0}$ and $h \leq h_{0}$, system (4.14)-(4.19) admits a unique solution $\left\{\Psi_{j}^{n}, \Phi^{n}, \Theta^{n}\right\}$, for $n=1,2, \cdots, N$, satisfying

$$
\begin{aligned}
& \left\|\Psi^{n}\right\|_{\infty}+\left|\Theta^{n}\right|+\left|\Phi^{n}\right| \leq K, \\
& \left\|e^{n}\right\|+\left|\varphi^{n}\right|+\left|\vartheta^{n}\right| \leq C^{*}\left(\tau+h^{2}\right) .
\end{aligned}
$$


Proof. At each discrete time $t=t_{n}$, the proposed numerical method produces a tridiagonal system of linear algebraic equations. Since the associated matrix is strictly diagonally dominant, then the numerical solution of the problem is unique.

Now, we prove the error estimates of the numerical scheme. Subtracting (4.14)-(4.19) from (4.5)-(4.10), we have the error equations: for $1 \leq n \leq N$

$$
\begin{aligned}
& \mathrm{i}{ }_{0}^{C} \mathbb{D}_{\tau}^{\alpha} e_{j}^{n}=- \delta_{x}^{2} e_{j}^{n}+V_{j} e_{j}^{n}+f\left(\left|\psi_{j}^{n-1}\right|^{2}\right) \psi_{j}^{n}-f\left(\left|\Psi_{j}^{n-1}\right|^{2}\right) \Psi_{j}^{n}+T_{j}^{n}, \quad 1 \leq j \leq J-1, \\
& \mathrm{i} \frac{h}{2}{ }_{0}{ }_{0} \mathbb{D}_{\tau}^{\alpha} e_{0}^{n}=-\delta_{x} e_{\frac{1}{2}}^{n}+\left(\vartheta^{n}+3 s_{0}^{\frac{\alpha}{2}} \mathbf{e}^{-\frac{\pi \mathrm{i}}{4}} e_{0}^{n}\right) \\
& \quad+\frac{h}{2} V_{0} e_{0}^{n}+\frac{h}{2}\left(f\left(\left|\psi_{0}^{n-1}\right|^{2}\right) \psi_{0}^{n}-f\left(\left|\Psi_{0}^{n-1}\right|^{2}\right) \Psi_{0}^{n}\right)+T_{0}^{n}, \\
&{ }_{0}^{C} \mathbb{D}_{\tau}^{\alpha} \vartheta^{n}=-\mathrm{i}\left(V_{0} \vartheta^{n}+f\left(\left|\psi_{0}^{n-1}\right|^{2}\right) \theta^{n}-f\left(\left|\Psi_{0}^{n-1}\right|^{2}\right) \Theta^{n}\right)-3 s_{0}^{\alpha} \vartheta^{n}-8 s_{0}^{3 \alpha / 2} \mathbf{e}^{-\frac{\pi \mathrm{i}}{4}} e_{0}^{n}+R_{0}^{n}, \\
& \mathrm{i} \frac{h}{2}{ }_{0}{ }_{0}^{C} \mathbb{D}_{\tau}^{\alpha} e_{J}^{n}= \delta_{x} e_{J-\frac{1}{2}}^{n}+\left(3 s_{0}^{\frac{\alpha}{2}} \mathbf{e}^{-\frac{\pi \mathrm{i}}{4}} e_{J}^{n}-\varphi^{n}\right) \\
& \quad+\frac{h}{2} V_{J} e_{J}^{n}+\frac{h}{2}\left(f\left(\left|\psi_{J}^{n-1}\right|^{2}\right) \psi_{J}^{n}-f\left(\left|\Psi_{J}^{n-1}\right|^{2}\right) \Psi_{J}^{n}\right)+T_{J}^{n}, \\
&{ }_{0}^{C} \mathbb{D}_{\tau}^{\alpha} \varphi^{n}=-\mathrm{i}\left[V_{J} \varphi^{n}+f\left(\left|\psi_{J}^{n-1}\right|^{2}\right) \phi^{n}-f\left(\left|\Psi_{J}^{n-1}\right|^{2}\right) \Phi^{n}\right]-3 s_{0}^{\alpha} \varphi^{n}+8 s_{0}^{\frac{3 \alpha}{2}} \mathbf{e}^{-\frac{\pi \mathrm{i}}{4}} e_{J}^{n}+R_{J}^{n}, \\
& e_{j}^{0}=0, \quad 0 \leq j \leq J .
\end{aligned}
$$

Multiplying Eq. (4.26) by $h \bar{e}_{i}^{n}$, and summing up over $j$ from 1 to $J-1$, we have

$$
\begin{aligned}
& \mathrm{i} h \sum_{j=1}^{J-1}\left({ }_{0}^{C} \mathbb{D}_{\tau}^{\alpha} e_{j}^{n}\right) \bar{e}_{j}^{n} \\
= & h \sum_{j=1}^{J-1}\left\{-\left(\delta_{x}^{2} e_{j}^{n}\right) \bar{e}_{j}^{n}+V_{j} e_{j}^{n} \bar{e}_{j}^{n}+\left[f\left(\left|\psi_{j}^{n-1}\right|^{2}\right) \psi_{j}^{n}-f\left(\left|\Psi_{j}^{n-1}\right|^{2}\right) \Psi_{j}^{n}\right] \bar{e}_{j}^{n}+T_{j}^{n} \bar{e}_{j}^{n}\right\} .
\end{aligned}
$$

Multiplying $\bar{e}_{0}^{n}, \mathrm{i} \bar{\vartheta}^{n}, \bar{e}_{J}^{n}$ and $\mathrm{i} \bar{\varphi}^{n}$ on both sides of (4.27)-(4.30), respectively, adding the results with the above formula, then using the following summation by parts formulas

$$
-h \sum_{j=1}^{J-1}\left(\delta_{x}^{2} e_{j}^{n}\right) \bar{e}_{j}^{n}-\bar{e}_{0}^{n} \delta_{x} e_{\frac{1}{2}}^{n}+\bar{e}_{J}^{n} \delta_{x} e_{J-\frac{1}{2}}^{n}=\left|e^{n}\right|_{1}^{2}
$$

and

$$
h\left(\frac{1}{2} \bar{e}_{0}^{n}{ }_{0}^{C} \mathbb{D}_{\tau}^{\alpha} e_{0}^{n}+\sum_{j=1}^{J-1}\left({ }_{0}^{C} \mathbb{D}_{\tau}^{\alpha} e_{j}^{n}\right) \bar{e}_{j}^{n}+\frac{1}{2} \bar{e}_{J}^{n}{ }_{0}^{C} \mathbb{D}_{\tau}^{\alpha} e_{J}^{n}\right)=\left({ }_{0}^{C} \mathbb{D}_{\tau}^{\alpha} e^{n}, e^{n}\right),
$$


we arrive at

$$
\begin{aligned}
& \mathrm{i}\left({ }_{0}^{C} \mathbb{D}_{\tau}^{\alpha} e^{n}, e^{n}\right)+i \bar{\varphi}^{n}{ }_{0}^{C} \mathbb{D}_{\tau}^{\alpha} \varphi^{n}+i \bar{\vartheta}^{n}{ }_{0}^{C} \mathbb{D}_{\tau}^{\alpha} \vartheta^{n} \\
= & \left|e^{n}\right|_{1}^{2}+h \sum_{j=1}^{J-1} V_{j} e_{j}^{n} \bar{e}_{j}^{n}+h \sum_{j=1}^{J-1}\left[f\left(\left|\psi_{j}^{n-1}\right|^{2}\right) \psi_{j}^{n}-f\left(\left|\Psi_{j}^{n-1}\right|^{2}\right) \Psi_{j}^{n}\right] \bar{e}_{j}^{n}+h \sum_{j=1}^{J-1} T_{j}^{n} \bar{e}_{j}^{n} \\
& +\left(\vartheta^{n}+3 s_{0}^{\frac{\alpha}{2}} \mathbf{e}^{-\frac{\pi}{4} i} e_{0}^{n}\right) \bar{e}_{0}^{n}+\frac{h}{2} V_{0} e_{0}^{n} \bar{e}_{0}^{n}+\frac{h}{2}\left(f\left(\left|\psi_{0}^{n-1}\right|^{2}\right) \psi_{0}^{n}-f\left(\left|\Psi_{0}^{n-1}\right|^{2}\right) \Psi_{0}^{n}\right) \bar{e}_{0}^{n}+T_{0}^{n} \bar{e}_{0}^{n} \\
& +\left(V_{0} \vartheta^{n}+f\left(\left|\psi_{0}^{n-1}\right|^{2}\right) \theta^{n}-f\left(\left|\Psi_{0}^{n-1}\right|^{2}\right) \Theta^{n}\right) \bar{\vartheta}^{n}-3 \mathrm{i} s_{0}^{\alpha} \vartheta^{n} \bar{\vartheta}^{n}-8 \mathrm{i} s_{0}^{3 \alpha / 2} \mathbf{e}^{-\frac{\pi \mathrm{i}}{4}} e_{0}^{n} \bar{\vartheta}^{n}+\mathrm{i} R_{0}^{n} \bar{\vartheta}^{n} \\
& +\left(3 s_{0}^{\frac{\alpha}{2}} \mathbf{e}^{-\frac{\pi \mathrm{i}}{4}} e_{J}^{n}-\varphi^{n}\right) \bar{e}_{J}^{n}+\frac{h}{2} V_{J} e_{J}^{n} \bar{e}_{J}^{n}+\frac{h}{2}\left(f\left(\left|\psi_{J}^{n-1}\right|^{2}\right) \psi_{J}^{n}-f\left(\left|\Psi_{J}^{n-1}\right|^{2}\right) \Psi_{J}^{n}\right) \bar{e}_{J}^{n}+T_{J}^{n} \bar{e}_{J}^{n} \\
& +\left[V_{J} \varphi^{n}+f\left(\left|\psi_{J}^{n-1}\right|^{2}\right) \phi^{n}-f\left(\left|\Psi_{J}^{n-1}\right|^{2}\right) \Phi^{n}\right] \bar{\varphi}^{n}-3 \mathrm{i} s_{0}^{\alpha} \varphi^{n} \bar{\varphi}^{n}+8 \mathrm{i} s_{0}^{\frac{3 \alpha}{2}} \mathbf{e}^{-\frac{\pi}{4} i} e_{J}^{n} \bar{\varphi}^{n}+R_{J}^{n} \bar{\varphi}^{n} .
\end{aligned}
$$

We now prove the results (4.24) and (4.25) by mathematical induction. First, we show that the estimates hold for $n=1$. Since $\left\{\Psi_{j}^{0}, \Phi^{0}, \Theta^{0}\right\}=\left\{\psi_{j}^{0}, \phi^{0}, \theta^{0}\right\}$, we have

$$
\begin{aligned}
& \left(f\left(\left|\psi_{j}^{0}\right|^{2}\right) \psi_{j}^{1}-f\left(\left|\Psi_{j}^{0}\right|^{2}\right) \Psi_{j}^{1}\right) \bar{e}_{j}^{1}=\left(f\left(\left|\psi_{j}^{0}\right|^{2}\right) \psi_{j}^{1}-f\left(\left|\psi_{j}^{0}\right|^{2}\right) \Psi_{j}^{1}\right) \bar{e}_{j}^{1}=f\left(\left|\psi_{j}^{0}\right|^{2}\right)\left|e_{j}^{1}\right|^{2}, \\
& \left(f\left(\left|\psi_{0}^{0}\right|^{2}\right) \theta^{1}-f\left(\left|\Psi_{0}^{0}\right|^{2}\right) \Theta^{1}\right) \bar{\vartheta}^{1}=\left(f\left(\left|\psi_{0}^{0}\right|^{2}\right) \theta^{1}-f\left(\left|\psi_{0}^{0}\right|^{2}\right) \Theta^{1}\right) \bar{\vartheta}^{1}=f\left(\left|\psi_{0}^{0}\right|^{2}\right)\left|\vartheta^{1}\right|^{2} \\
& \left(f\left(\left|\psi_{J}^{0}\right|^{2}\right) \phi^{1}-f\left(\left|\Psi_{J}^{0}\right|^{2}\right) \Phi^{1}\right) \bar{\varphi}^{1}=\left(f\left(\left|\psi_{J}^{0}\right|^{2}\right) \phi^{1}-f\left(\left|\psi_{J}^{0}\right|^{2}\right) \Phi^{1}\right) \bar{\varphi}^{1}=f\left(\left|\psi_{J}^{0}\right|^{2}\right)\left|\varphi^{1}\right|^{2}
\end{aligned}
$$

Moreover, it holds that

$$
\begin{aligned}
& \operatorname{Im}\left(\left(\vartheta^{1}+3 s_{0}^{\frac{\alpha}{2}} \mathbf{e}^{-\frac{\pi}{4} i} e_{0}^{1}\right) \bar{e}_{0}^{1}+T_{0}^{1} \bar{e}_{0}^{1}\right) \\
= & -\frac{3 \sqrt{2}}{2} 3 s_{0}^{\frac{\alpha}{2}}\left|e_{0}^{1}\right|^{2}+\operatorname{Im}\left(\vartheta^{1} \bar{e}_{0}^{1}+T_{0}^{1} \bar{e}_{0}^{1}\right) \\
\leq & -\frac{3 \sqrt{2}}{2} 3 s_{0}^{\frac{\alpha}{2}}\left|e_{0}^{1}\right|^{2}+\varepsilon_{1}\left|e_{0}^{1}\right|^{2}+\frac{1}{4 \varepsilon_{1}}\left|\vartheta^{1}\right|^{2}+\left(\frac{3 \sqrt{2}}{2} 3 s_{0}^{\frac{\alpha}{2}}-\varepsilon_{1}\right)\left|e_{0}^{1}\right|^{2}+\frac{1}{4\left(\frac{3 \sqrt{2}}{2} 3 s_{0}^{\frac{\alpha}{2}}-\varepsilon_{1}\right)}\left|T_{0}^{1}\right|^{2} \\
= & \frac{1}{4 \varepsilon_{1}}\left|\vartheta^{1}\right|^{2}+\frac{1}{4\left(\frac{3 \sqrt{2}}{2} 3 s_{0}^{\frac{\alpha}{2}}-\varepsilon_{1}\right)}\left|T_{0}^{1}\right|^{2},
\end{aligned}
$$

where $\varepsilon_{1}$ is a constant, satisfying $0<\varepsilon_{1}<\frac{3 \sqrt{2}}{2} 3 s_{0}^{\frac{\alpha}{2}}$. Similarly, we have

$$
\operatorname{Im}\left(\left(3 s_{0}^{\frac{\alpha}{2}} \mathbf{e}^{-\frac{\pi \mathrm{i}}{4}} e_{J}^{1}-\varphi^{1}\right) \bar{e}_{J}^{1}+T_{J}^{1} \bar{e}_{J}^{1}\right) \leq \frac{1}{4 \varepsilon_{1}}\left|\varphi^{1}\right|^{2}+\frac{1}{4\left(\frac{3 \sqrt{2}}{2} 3 s_{0}^{\frac{\alpha}{2}}-\varepsilon_{1}\right)}\left|T_{J}^{1}\right|^{2} .
$$

Thus, taking the imaginary part of (4.32) and using the Cauchy-Schwarz inequality yields

$$
\operatorname{Re}\left(\left({ }_{0}^{C} \mathbb{D}_{\tau}^{\alpha} e^{1}, e^{1}\right)+\bar{\varphi}^{1}{ }_{0}^{C} \mathbb{D}_{\tau}^{\alpha} \varphi^{1}+\bar{\vartheta}^{1}{ }_{0}^{C} \mathbb{D}_{\tau}^{\alpha} \vartheta^{1}\right) \leq C\left\|e^{1}\right\|^{2}+C\left|\varphi^{1}\right|^{2}+C\left|\vartheta^{1}\right|^{2}+C\left(\tau+h^{2}\right)^{2} .
$$


By Lemma 4.3, we conclude that there exists a parameter $\tau_{1}$ such that, for $\tau \leq \tau_{1}$,

$$
\left\|e^{1}\right\|+\left|\varphi^{1}\right|+\left|\vartheta^{1}\right| \leq C^{*}\left(\tau+h^{2}\right) .
$$

Meanwhile, we have

$$
\left|e^{1}\right|_{1}^{2}=h \sum_{j=1}^{J}\left|\delta_{x} \bar{e}_{j-\frac{1}{2}}^{1}\right|\left|\delta_{x} e_{j-\frac{1}{2}}^{1}\right| \leq h \sum_{j=1}^{J}\left(\frac{\left|e_{j}^{1}\right|+\left|e_{j-1}^{1}\right|}{h}\right)^{2} \leq \frac{4}{h^{2}}\left\|e^{1}\right\|^{2} \leq \frac{4 C^{*}}{h^{2}}\left(\tau+h^{2}\right)^{2} .
$$

Together with (4.39), (4.40) and Lemma 4.1, we deduce that

$$
\left\|e^{1}\right\|_{L^{\infty}} \leq C\left(\tau+h^{2}+\frac{\tau}{h}\right) .
$$

Hence, one obtains

$$
\begin{aligned}
\left\|\Psi^{1}\right\|_{\infty}+\left|\Theta^{1}\right|+\left|\Phi^{1}\right| & \leq\left\|\psi^{1}\right\|_{\infty}+\left|\theta^{1}\right|+\left|\phi^{1}\right|+\left\|e^{1}\right\|_{L^{\infty}}+\left|\vartheta^{1}\right|+\left|\vartheta^{1}\right| \\
& \leq\left\|\psi^{1}\right\|_{\infty}+\left|\theta^{1}\right|+\left|\phi^{1}\right|+C_{1}\left(\tau+h^{2}+\frac{\tau}{h}\right) \leq K,
\end{aligned}
$$

whenever $C_{1}\left(\tau+h^{2}+\frac{\tau}{h}\right) \leq 1$.

Now, suppose that the main results (4.24) and (4.25) hold for $n \leq k-1$, then we have

$$
\begin{aligned}
& \left(f\left(\left|\psi_{j}^{k-1}\right|^{2}\right) \psi_{j}^{k}-f\left(\left|\Psi_{j}^{k-1}\right|^{2}\right) \Psi_{j}^{k}\right) \\
= & \left(f\left(\left|\psi_{j}^{k-1}\right|^{2}\right) \psi_{j}^{k}-f\left(\left|\Psi_{j}^{k-1}\right|^{2}\right) \psi_{j}^{k}\right)+\left(f\left(\left|\Psi_{j}^{k-1}\right|^{2}\right) \psi_{j}^{k}-f\left(\left|\Psi_{j}^{k-1}\right|^{2}\right) \Psi_{j}^{k}\right) \\
= & \psi_{j}^{k} f^{\prime}\left(\xi_{j}^{k-1}\right)\left(\left|\psi_{j}^{k-1}\right|^{2}-\left|\Psi_{j}^{k-1}\right|^{2}\right)+f\left(\left|\Psi_{j}^{k-1}\right|^{2}\right)\left(\psi_{j}^{k}-\Psi_{j}^{k}\right) \\
\leq & C\left(\left|e_{j}^{k-1}\right|+\left|e_{j}^{k}\right|\right),
\end{aligned}
$$

where $\xi_{j}^{k-1} \in\left(\psi_{j}^{k-1}, \Psi_{j}^{k-1}\right)$ and we remark that $\left\|\Psi_{j}^{k-1}\right\|_{L^{\infty}} \leq K$. Similarly, we show that

$$
\begin{aligned}
& f\left(\left|\psi_{0}^{k-1}\right|^{2}\right) \theta^{k}-f\left(\left|\Psi_{0}^{k-1}\right|^{2}\right) \Theta^{k} \leq C\left|e_{0}^{k-1}\right|+C\left|\vartheta^{k}\right|, \\
& f\left(\left|\psi_{J}^{k-1}\right|^{2}\right) \phi^{k}-f\left(\left|\Psi_{J}^{k-1}\right|^{2}\right) \Phi^{k} \leq C\left|e_{J}^{k-1}\right|+C\left|\varphi^{k}\right| .
\end{aligned}
$$

In addition, we can write that

$$
\begin{aligned}
& \operatorname{Im}\left(\left(\vartheta^{k}+3 s_{0}^{\frac{\alpha}{2}} \mathbf{e}^{-\frac{\pi}{4} i} e_{0}^{k}\right) \bar{e}_{0}^{k}+T_{0}^{k} \bar{e}_{0}^{k}-8 \mathrm{i} s_{0}^{3 \alpha / 2} \mathbf{e}^{-\frac{\pi \mathrm{i}}{4}} e_{0}^{k} \bar{\vartheta}^{k}\right) \\
= & -\frac{3 \sqrt{2}}{2} s_{0}^{\frac{\alpha}{2}}\left|e_{0}^{k}\right|^{2}+\operatorname{Im}\left(\vartheta^{k} \bar{e}_{0}^{k}+T_{0}^{k} \bar{e}_{0}^{n}-8 \mathrm{i} s_{0}^{3 \alpha / 2} \mathbf{e}^{-\frac{\pi \mathrm{i}}{4}} e_{0}^{k} \bar{\vartheta}^{k}\right) \\
\leq & -\frac{3 \sqrt{2}}{2} s_{0}^{\frac{\alpha}{2}}\left|e_{0}^{k}\right|^{2}+\left(C_{1}\left|\bar{e}_{0}^{k}\right|^{2}+\frac{1}{4 C_{1}}\left|\vartheta^{k}\right|^{2}\right)+\left(C_{2}\left|\bar{e}_{0}^{k}\right|^{2}+\frac{1}{4 C_{2}}\left|T_{0}^{k}\right|^{2}\right) \\
& +8 s_{0}^{3 \alpha / 2}\left(C_{3}\left|\bar{e}_{0}^{k}\right|^{2}+\frac{1}{4 C_{4}}\left|\vartheta^{k}\right|^{2}\right),
\end{aligned}
$$


where $C_{i}>0, i=1,2,3$ are arbitrary positive and small constants. Let us set $C_{1}+C_{2}+$ $8 s_{0}^{3 \alpha / 2} C_{3}=3 \sqrt{2} s_{0}^{\frac{\alpha}{2}} / 2$ in the above formula. We then deduce that

$$
\operatorname{Im}\left(\left(\vartheta^{k}+3 s_{0}^{\frac{\alpha}{2}} \mathbf{e}^{-\frac{\pi}{4} i} e_{0}^{k}\right) \bar{e}_{0}^{k}+T_{0}^{k} \bar{e}_{0}^{k}-8 \mathrm{i} s_{0}^{3 \alpha / 2} \mathbf{e}^{-\frac{\pi \mathrm{i}}{4}} e_{0}^{k} \bar{\vartheta}^{k}\right) \leq C\left|\vartheta^{k}\right|^{2}+C\left|T_{0}^{k}\right|^{2}
$$

Similarly to (4.44), we obtain

$$
\operatorname{Im}\left(\left(3 s_{0}^{\frac{\alpha}{2}} \mathbf{e}^{-\frac{\pi \mathrm{i}}{4}} e_{J}^{n}-\varphi^{n}\right) \bar{e}_{J}^{n}+T_{J}^{n} \bar{e}_{J}^{n}+8 \mathrm{i} s_{0}^{\frac{3 \alpha}{2}} \mathbf{e}^{-\frac{\pi}{4} i} e_{J}^{n} \bar{\varphi}^{n}\right) \leq C\left|\varphi^{n}\right|^{2}+C\left|T_{J}^{n}\right|^{2} .
$$

Now, let us consider $n=k$ in (4.32). By taking the imaginary part of the resulting equation, using (4.41)-(4.45) and the Cauchy-Schwarz inequality, one gets

$$
\begin{array}{rl} 
& \operatorname{Re}\left(\left({ }_{0}^{C} \mathbb{D}_{\tau}^{\alpha} e^{k}, e^{k}\right)+\bar{\varphi}^{k}{ }_{0}^{C} \mathbb{D}_{\tau}^{\alpha} \varphi^{k}+\bar{\vartheta}^{k}{ }_{0}^{C} \mathbb{D}_{\tau}^{\alpha} \vartheta^{k}\right) \\
\leq C & C\left(\left\|e^{k-1}\right\|^{2}+\left\|e^{k}\right\|^{2}+\left|\varphi^{k}\right|^{2}+\left|\vartheta^{k}\right|^{2}+\left(\tau+h^{2}\right)^{2}\right) .
\end{array}
$$

Now, by Lemma 4.3, when $\tau<\tau_{2}$, we prove that there exists a parameter $\tau_{2}$ such that

$$
\left\|e^{k}\right\|+\left|\varphi^{k}\right|+\left|\vartheta^{k}\right| \leq C^{*}\left(\tau+h^{2}\right) .
$$

Moreover, we have

$$
\left|e^{k}\right|_{1}^{2}=h\left|\sum_{j=1}^{J}\left(\delta_{x} \bar{e}_{j-\frac{1}{2}}^{k}\right)\left(\delta_{x} e_{j-\frac{1}{2}}^{k}\right)\right| \leq \frac{4}{h^{2}}\left\|e^{k}\right\|^{2} \leq \frac{4 C^{*}}{h^{2}}\left(\tau+h^{2}\right)^{2} .
$$

Together with (4.47), (4.48) and Lemma 4.1, we deduce that

$$
\left\|e^{k}\right\|_{L^{\infty}} \leq C\left(\tau+h^{2}+\frac{\tau}{h}\right) .
$$

Therefore, we obtain

$$
\begin{aligned}
\left\|\Psi^{k}\right\|_{\infty}+\left|\Theta^{k}\right|+\left|\Phi^{k}\right| & \leq\left\|\psi^{k}\right\|_{\infty}+\left|\theta^{k}\right|+\left|\phi^{k}\right|+\left\|e^{k}\right\|_{L^{\infty}+\left|\vartheta^{k}\right|+\left|\vartheta^{k}\right|} \\
& \leq\left\|\psi^{k}\right\|_{\infty}+\left|\theta^{k}\right|+\left|\phi^{k}\right|+C\left(\tau+h^{2}+\frac{\tau}{h}\right) \leq K,
\end{aligned}
$$

as long as $C_{2}\left(\tau+h^{2}+\frac{\tau}{h}\right) \leq 1$. Finally, the conclusions also hold for $n=k$, completing hence the proof.

\subsection{Fast evaluation based on the L1-scheme}

In this section, we consider the fast evaluation of the Caputo derivative, proposed in [20], to circumvent the huge storage and computational cost for the long time simulation. The 
main idea of this fast algorithm is to split the Caputo derivative into the sum of a local part and a history part

$$
{ }_{0}^{C} \mathcal{D}_{t}^{\alpha} \psi^{n}=\frac{1}{\Gamma(1-\alpha)} \int_{t_{n-1}}^{t_{n}} \frac{\psi^{\prime}(x, s) d s}{\left(t_{n}-s\right)^{\alpha}}+\frac{1}{\Gamma(1-\alpha)} \int_{0}^{t_{n-1}} \frac{\psi^{\prime}(s) d s}{\left(t_{n}-s\right)^{\alpha}}:=C_{\text {loc }}\left(t_{n}\right)+C_{\text {hist }}\left(t_{n}\right) .
$$

For the local part $C_{\mathrm{loc}}\left(t_{n}\right)$, we apply the L1-approximation, i.e.,

$$
C_{\mathrm{loc}}\left(t_{n}\right) \approx \frac{\psi\left(x, t_{n}\right)-\psi\left(x, t_{n-1}\right)}{\tau \Gamma(1-\alpha)} \int_{t_{n-1}}^{t_{n}} \frac{1}{\left(t_{n}-s\right)^{\alpha}} d s=\frac{\psi\left(x, t_{n}\right)-\psi\left(x, t_{n-1}\right)}{\tau^{\alpha} \Gamma(2-\alpha)} .
$$

For the history part $C_{\text {hist }}\left(t_{n}\right)$, we integrate by part and get

$$
C_{\text {hist }}\left(t_{n}\right)=\frac{1}{\Gamma(1-\alpha)}\left[\frac{\psi\left(x, t_{n-1}\right)}{\tau^{\alpha}}-\frac{\psi\left(x, t_{0}\right)}{t_{n}^{\alpha}}-\alpha \int_{0}^{t_{n-1}} \frac{\psi(x, s) d s}{\left(t_{n}-s\right)^{1+\alpha}}\right] .
$$

Then, we use a sum-of-exponentials expansion to approximate the convolution integral of $\psi^{n}$ with the kernel $t^{-1-\alpha}$. For a given absolute error $\varepsilon$ and for $\alpha$, there exist some positive real numbers $s_{i}$ and $w_{i}, i=1, \cdots, N_{\exp }$ ( $N_{\exp }$ is the number of exponentials) such that

$$
\left|\frac{1}{t^{1+\alpha}}-\sum_{i=1}^{N_{\exp }} \omega_{i} e^{-s_{i} t}\right| \leq \varepsilon, \quad \text { for all } t \in[\tau, T] .
$$

We replace the kernel $\frac{1}{t^{1+\alpha}}$ in (4.50) by its sum-of-exponentials approximation in (4.51) to have

$$
C_{\text {hist }}\left(t_{n}\right) \approx \frac{1}{\Gamma(1-\alpha)}\left[\frac{\psi\left(x, t_{n-1}\right)}{\tau^{\alpha}}-\frac{\psi\left(x, t_{0}\right)}{t_{n}^{\alpha}}-\alpha \sum_{i=1}^{N_{\text {exp }}} \omega_{i} U_{\text {hist }, i}\left(t_{n}\right)\right],
$$

where $U_{\text {hist }, i}\left(t_{n}\right)$ is defined by

$$
U_{\text {hist }, i}\left(t_{n}\right)=\int_{0}^{t_{n-1}} e^{-\left(t_{n}-s\right) s_{i}} \psi(x, s) d s,
$$

and has a simple recurrence relation

$$
U_{\text {hist }, i}\left(t_{n}\right)=e^{-s_{i} \tau} U_{\text {hist }, i}\left(t_{n-1}\right)+\int_{t_{n-2}}^{t_{n-1}} e^{-s_{i}\left(t_{n}-s\right)} \psi(x, s) d s,
$$

with $U_{\text {hist }, i}\left(t_{0}\right)=0$. The integral can be calculated by

$$
\int_{t_{n-2}}^{t_{n-1}} e^{-s_{i}\left(t_{n}-s\right)} \psi(x, s) d s \approx \frac{e^{-s_{i} \tau}}{s_{i}^{2} \tau}\left[\left(e^{-s_{i} \tau}-1+s_{i} \tau\right) \psi^{n-1}+\left(1-e^{-s_{i} \tau}-e^{-s_{i} \tau} s_{i} \tau\right) \psi^{n-2}\right] .
$$


Finally, the Fast approximate evaluation of the Caputo fractional derivative is given by

$$
{ }_{0}^{F C} \mathbb{D}_{t}^{\alpha} \psi^{n}=\frac{\psi\left(x, t_{n}\right)-\psi\left(x, t_{n-1}\right)}{\tau^{\alpha} \Gamma(2-\alpha)}+\frac{1}{\Gamma(1-\alpha)}\left[\frac{\psi\left(x, t_{n-1}\right)}{\tau^{\alpha}}-\frac{\psi\left(x, t_{0}\right)}{t_{n}^{\alpha}}-\alpha \sum_{i=1}^{N_{\text {exp }}} \omega_{i} U_{\text {hist }, i}\left(t_{n}\right)\right],
$$

for $n>0$, and where $U_{\text {hist, } i}\left(t_{n}\right)$ can be obtained by the recurrence relation (4.52).

By using the fast evaluation of the Caputo derivative instead of the direct L1approximation in (4.14)-(4.19), we have the finite difference scheme given by

$$
\begin{aligned}
& \mathrm{i}_{0}^{F C} \mathbb{D}_{t}^{\alpha} \Psi_{j}^{n}=-\delta_{x}^{2} \Psi_{j}^{n}+\left[V_{j}+f\left(\left|\Psi_{j}^{n-1}\right|^{2}\right)\right] \Psi_{j}^{n}, \quad 1 \leq j \leq J-1, \quad 1 \leq n \leq N, \\
& \mathrm{i} \frac{h}{2}{ }^{F C} \mathbb{D}_{t}^{\alpha} \Psi_{0}^{n}=-\delta_{x} \Psi_{\frac{1}{2}}^{n}+\left(\Theta^{n}-3 s_{0}^{\frac{\alpha}{2}} \mathbf{e}^{-\frac{\pi \mathrm{i}}{4}} \Psi_{0}^{n}\right)+\frac{h}{2}\left[V_{0}+f\left(\left|\Psi_{0}^{n-1}\right|^{2}\right)\right] \Psi_{0}^{n}, \\
& { }_{0}^{F C} \mathbb{D}_{t}^{\alpha} \Theta^{n}=-\mathrm{i}\left[V_{0}+f\left(\left|\Psi_{0}^{n-1}\right|^{2}\right)\right] \Theta^{n}+3 s_{0}^{\alpha} \Theta^{n}+8 s_{0}^{\frac{3 \alpha}{2}} \mathbf{e}^{-\frac{\pi \mathrm{i}}{4}} \Psi_{0}^{n}, \\
& \mathrm{i} \frac{h_{2}}{{ }^{F C}} \mathbb{D}_{t}^{\alpha} \Psi_{J}^{n}=\delta_{x} \Psi_{J-\frac{1}{2}}^{n}+\left(3 s_{0}^{\frac{\alpha}{2}} \mathbf{e}^{-\frac{\pi \mathrm{i}}{4}} \Psi_{J}^{n}-\Phi^{n}\right)+\frac{h}{2}\left[V_{J}+f\left(\left|\Psi_{J}^{n-1}\right|^{2}\right)\right] \Psi_{J}^{n}, \\
& { }_{0}^{F C} \mathbb{D}_{t}^{\alpha} \Phi^{n}=-\mathrm{i}\left[V_{J}+f\left(\left|\Psi_{J}^{n-1}\right|^{2}\right)\right] \Phi^{n}+3 s_{0}^{\alpha} \Phi^{n}-8 s_{0}^{\frac{3 \alpha}{2}} \mathbf{e}^{-\frac{\pi \mathrm{i}}{4}} \Psi_{J}^{n}, \\
& \Psi_{j}^{0}=\Psi_{0}\left(x_{j}\right), \quad 0 \leq j \leq J .
\end{aligned}
$$

\section{Numerical examples}

In this section, we provide four examples to demonstrate the effectiveness of our method, and illustrate the dynamics of numerical solutions of TFNSEs in different situations.

Example 5.1. In this example we consider the TFNSE with cubic nonlinearity, i.e. $f(|\psi|)=$ $2|\psi|^{2}$ and with the gaussian potential $V(x):=\mathbf{e}^{-5 x^{2}}$. The initial data is: $\psi_{0}(x)=\mathbf{e}^{-5 x^{2}}$. For the calculations in Figs. 1 and 2, we fix: $T=4$ and $] x_{\ell}, x_{r}[:=]-5,5[$. The left and right pictures represent the amplitude of the wave field, i.e. $\left|\psi_{\tau, h}^{\alpha, \text { fast }}\right|$, for $\alpha=0.5$ and $\alpha=$ 0.75, respectively, and computed by the fast scheme (4.54)-(4.59) for the discretization parameters $\tau:=T / N=10^{-3}\left(N=4 \times 10^{3}\right)$ and $h:=\left(x_{r}-x_{\ell}\right) / J=5 \times 10^{-2}(J=200)$, with the tolerance parameter $\varepsilon=10^{-9}$. For the ABCs, we fix $s_{0}=20$ (we see below that any other value $s_{0}$ does not modify the results). The CPU time for $\alpha=0.5$ is 21 (sec.) and 27 (sec.) for $\alpha=0.75$. Using the fast scheme compared with the direct scheme (4.14)-(4.19) does not affect the accuracy. Indeed, we report on Fig. 2 the absolute error between $\psi_{\tau, h}^{\alpha, \text { fast }}$ and $\psi_{\tau, h}^{\alpha, \text { dir }}$ ("dir" means "direct" here): $\left|\psi_{\tau, h}^{\alpha, \text { fast }}-\psi_{\tau, h}^{\alpha, \text { dir }}\right|$, on the grid for the same discretization parameters $\tau$ and $h$, and the two values of $\alpha$. The error is smaller than $\varepsilon$ and clearly does not modify the accuracy of a given computation. To demonstrate the complexity of the two schemes (direct vs. fast), we plot on Fig. 3 the CPU time of the two schemes in seconds vs. the number $N$ of grid points in time. We observe that while the CPU time for 

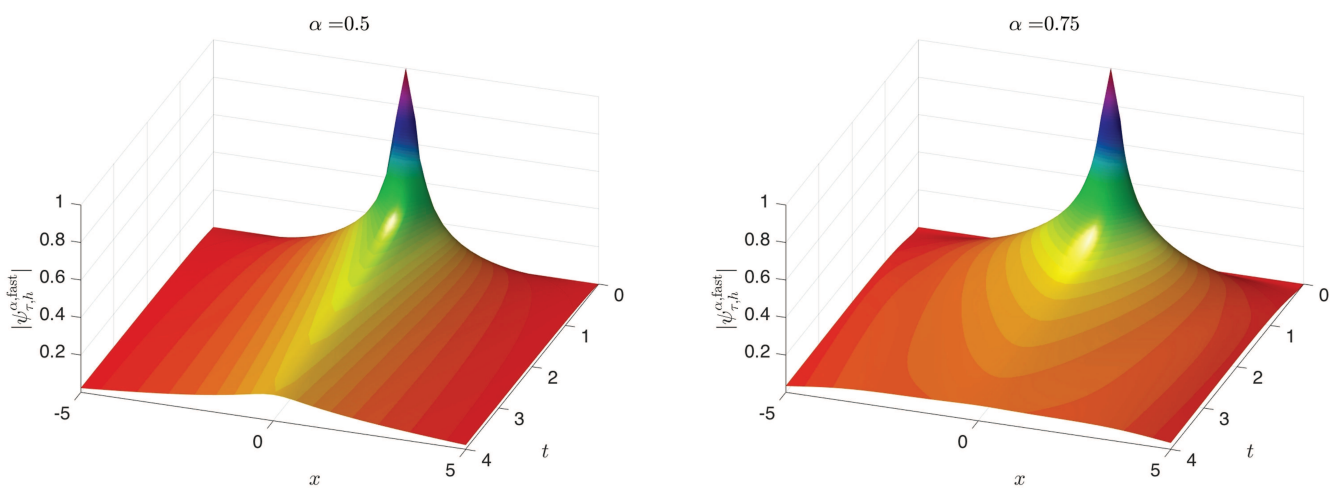

Figure 1: Example 5.1: Amplitude $\left|\psi_{\tau, h}^{\alpha, \text { fast }}\right|$ for $\alpha=0.5$ (left) and $\alpha=0.75$ (right).
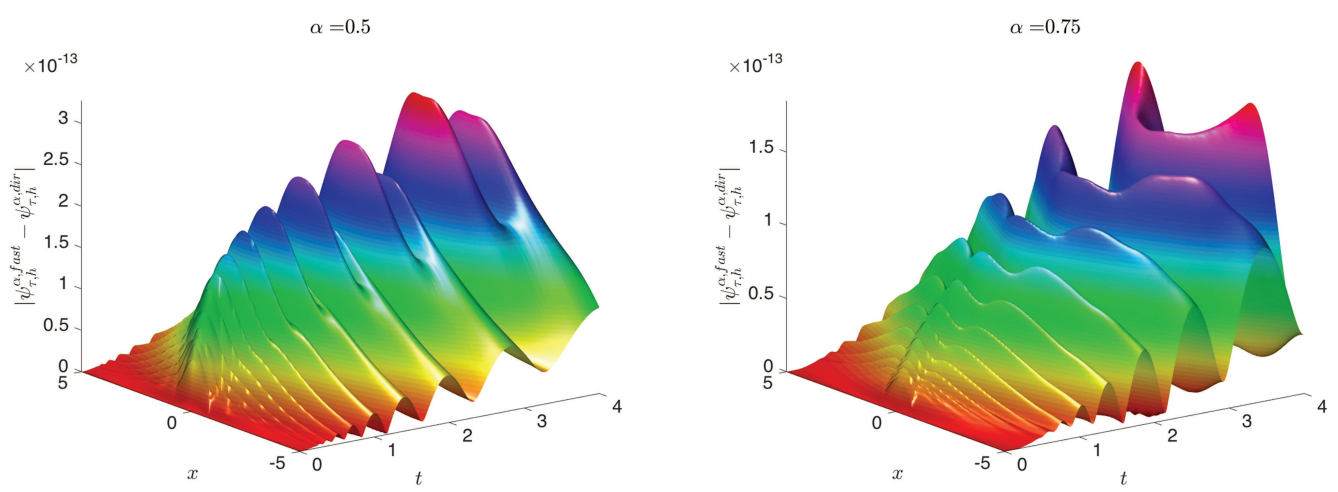

Figure 2: Example 5.1: Absolute error $\left|\psi_{\tau, h}^{\alpha, \text { fast }}-\psi_{\tau, h}^{\alpha, \text { dir }}\right|$ for $\alpha=0.5$ (left) and $\alpha=0.75$ (right).
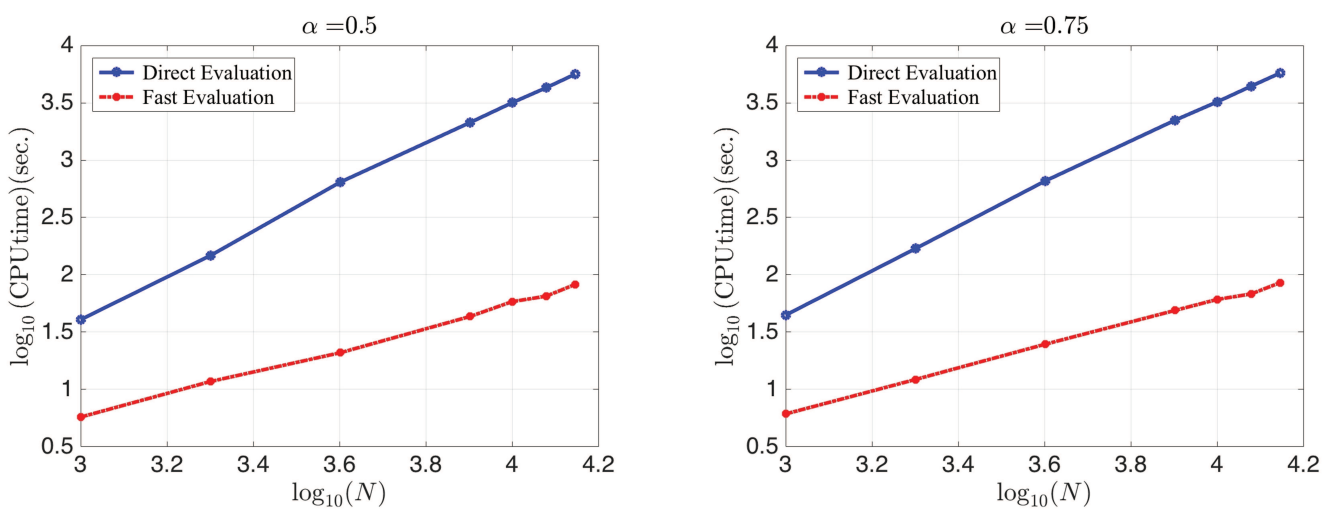

Figure 3: Example 5.1: Comparison of the CPU time (sec. in $\log _{10}$-scale) required for the direct and fast methods vs. the number $N$ of time points $\left(\log _{10}\right.$ scale) (left: $\alpha=0.5$; right: $\left.\alpha=0.75\right)$. 

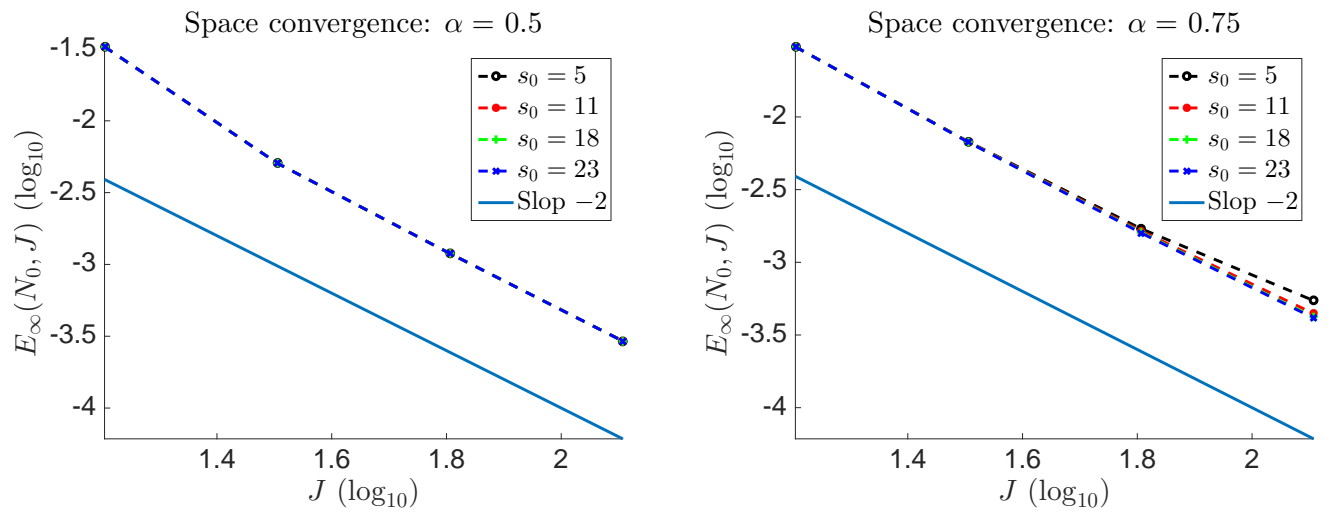

Figure 4: Example 5.1: $E_{\infty}\left(N_{0}, J\right)$ vs. $J$ (both in $\log _{10}$-scale) for various values of $s_{0}$ and by using the fast scheme (4.54)-(4.59) (left: $\alpha=0.5$; right: $\alpha=0.75)$.

the direct scheme scales as $\mathcal{O}\left(N^{2}\right)$, it increases almost linearly with respect to $N$ for the fast scheme. There is a significant speed-up even for moderate values of $N$.

From these first computations, we conclude that the fast scheme leads to a similar accuracy as for the direct scheme (according to the tolerance $\varepsilon$, other computations confirming this observation) while being much more efficient, with a nearly linear cost in time for a low storage, most particularly when $\tau$ gets smaller. For these reasons, we now always use the fast scheme to study the convergence rates in space and time, and for the various simulations. We also denote $\psi_{\tau, h}^{\alpha, \text { fast }}$ by $\psi_{\tau, h}$ to simplify the notations.

We now analyze both the truncation error related to the introduction of an $A B C$ and the convergence rate of the fast scheme. To this aim, we compute a reference solution $\psi^{\text {ref }}$ on the larger domain ] $-12,12$ [ until the time $T=0.5$, for some extremely small values of $\tau$ and $h$. We report the following error: we define $E_{\infty}(N, J):=|| \psi^{\text {ref }}-\psi_{\tau, h} \|_{\infty}$ for two fixed values of $\tau$ and $h$. When analyzing the convergence rate in space of the fast scheme, we represent the quantity $E_{\infty}\left(N_{0}, J\right):=|| \psi^{\text {ref }}-\psi_{\tau_{0}, h} \|_{\infty}$ vs. $J$ (both in $\log _{10^{-s c a l e}}$ ) for $\tau_{0}=10^{-4}$ $\left(N_{0}=5 \times 10^{3}\right)$ and for various values of $s_{0}$. When we study the convergence rate in time, we fix $h_{0}:=5 \times 10^{-3}\left(J_{0}=2 \times 10^{3}\right)$ and represent $E_{\infty}\left(N, J_{0}\right):=\left\|\psi^{\text {ref }}-\psi_{\tau, h_{0}}\right\|_{\infty}$ vs. $N\left(\right.$ in $\log _{10^{-}}$ scale). From Figs. 4 and 5, we show that both the convergence rate of the fast scheme (4.54)-(4.59) (as well as direct scheme (4.14)-(4.19)) is of the order of $\mathcal{O}\left(h^{2}+\tau\right)$. In addition, the ABCs are accurate and not affected by the choice of the parameter $s_{0}$. Finally, an analysis (not reported here) shows that the $L_{\infty}$-error of the direct and fast schemes is almost the same (up to the tolerance $\varepsilon$ ).

Example 5.2. In this second example, we investigate the evolution of the numerical solution $\psi$ of the TFNSE for different fractional orders $\alpha$ with the nonlinearity $f\left(|\psi|^{2}\right) \psi=$ $-2|\psi|^{2} \psi$ and for $V(x)=0$. The initial data is $\psi_{0}=\mathbf{e}^{2 \mathrm{i}(x+3)} \operatorname{sech}(x+3)$. The computational domain is ] $-10,10$ [ for a maximal time of computation $T=3$. The discretization parameters are $\tau=3 \times 10^{-3}\left(N=10^{3}\right)$ and $h=10^{-3}\left(J=2 \times 10^{3}\right)$. For the ABC, we consider $s_{0}=20$. 

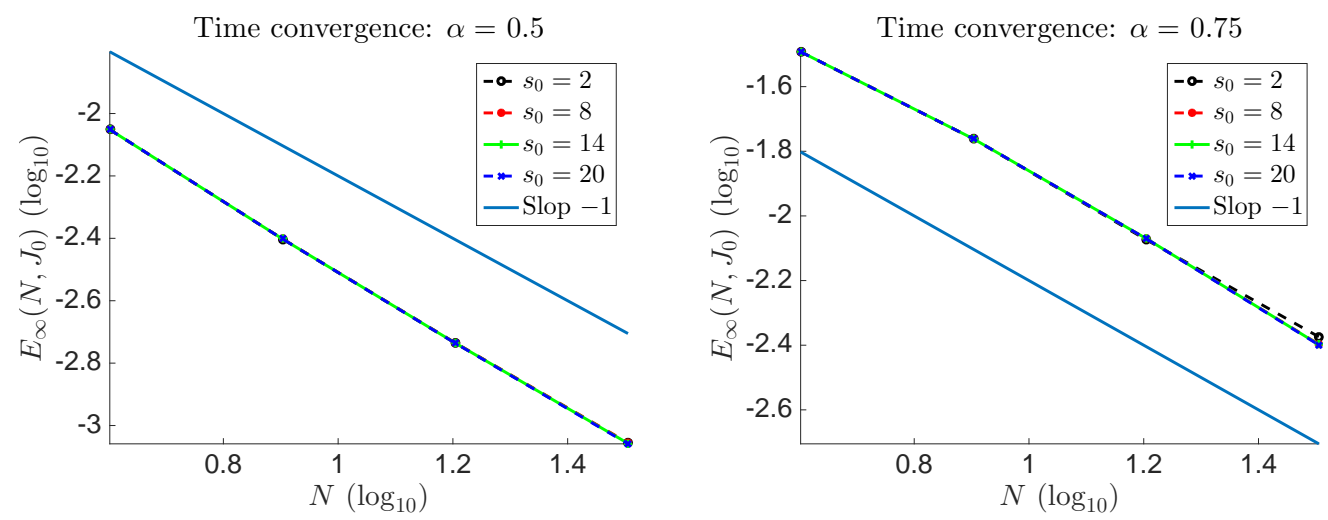

Figure 5: Example 5.1: $E_{\infty}\left(N, J_{0}\right)$ vs. $N$ (both in $\log _{10}$-scale) for various values of $s_{0}$ and by using the fast scheme (4.54)-(4.59) (left: $\alpha=0.5$; right: $\alpha=0.75)$.

In the standard situation, i.e. for $\alpha=1$, the soliton propagates from the left to the right domain with a given angle. One can see on Fig. 6 that the evolution of the solution is pretty sharp on the first time steps and then the solution propagates straight when $\alpha=0.25$ with less dispersion. The situation tends to the standard case when $\alpha$ tends to 1 .
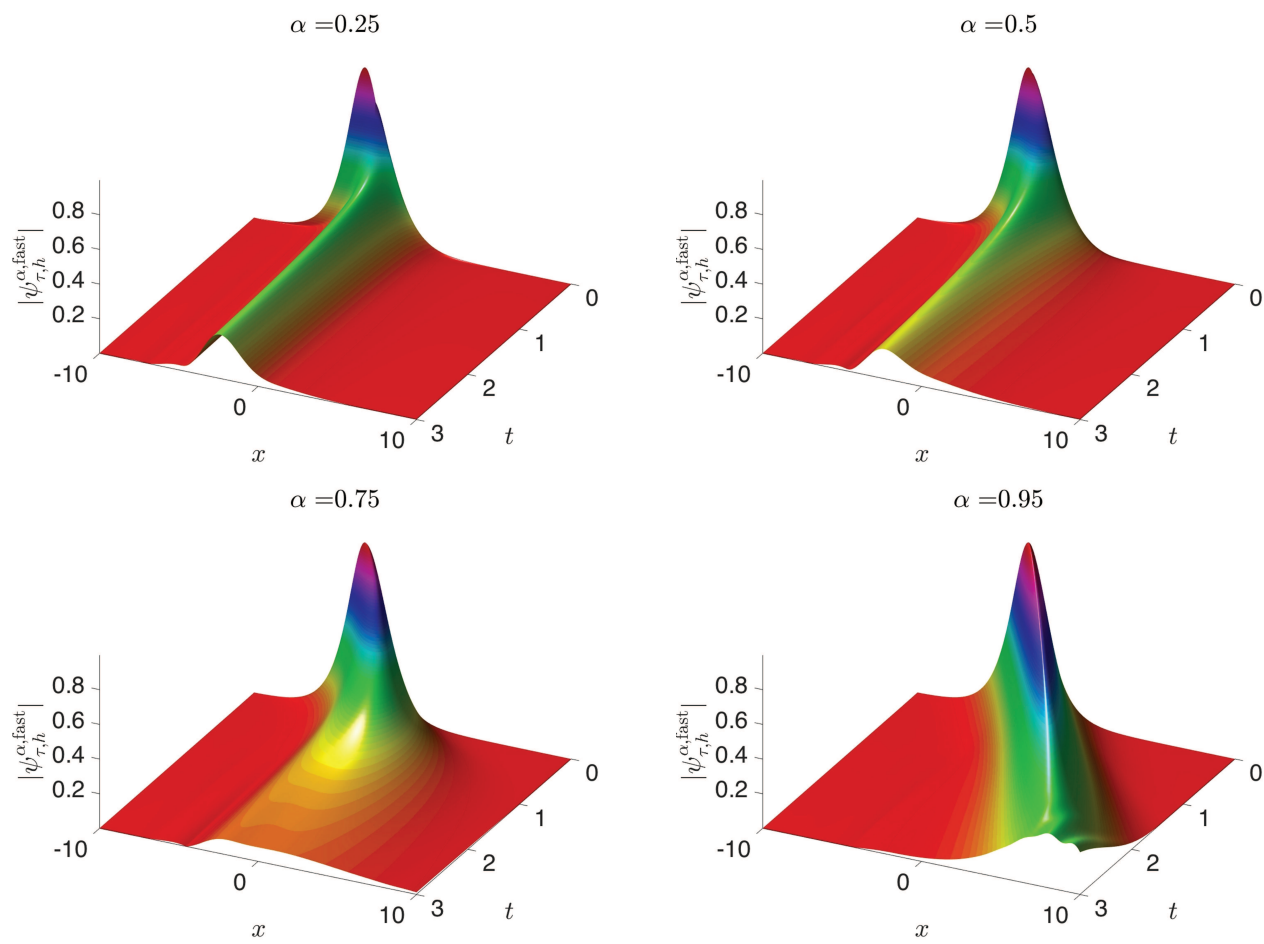

Figure 6: Example 5.2: Evolution of $|\psi|$ for $\alpha=0.25,0.5,0.75$ and 0.95. 
Example 5.3. This third example, reported on Fig. 7, is dedicated to the collision of two waves for different values of $\alpha$. The nonlinearity and the external potential for the TFNSE are respectively given by

$$
f\left(|\psi|^{2}\right) \psi=-2|\psi|^{2} \psi, \quad V(x)=\mathbf{e}^{-3 x^{2}} .
$$

The initial data is built as the superposition of two waves

$$
\psi_{0}(x)=\mathbf{e}^{-2 \mathrm{i}(x-8)} \operatorname{sech}(x-8)+\mathbf{e}^{2 \mathrm{i}(x+8)} \operatorname{sech}(x+8) .
$$

We consider the interval ] $-20,20$ [ and a final computational time $T=7$. We fix $N=4000$ grid points in time and $J=1000$ spatial discrete points. For the ABC, we choose $s_{0}=20$.
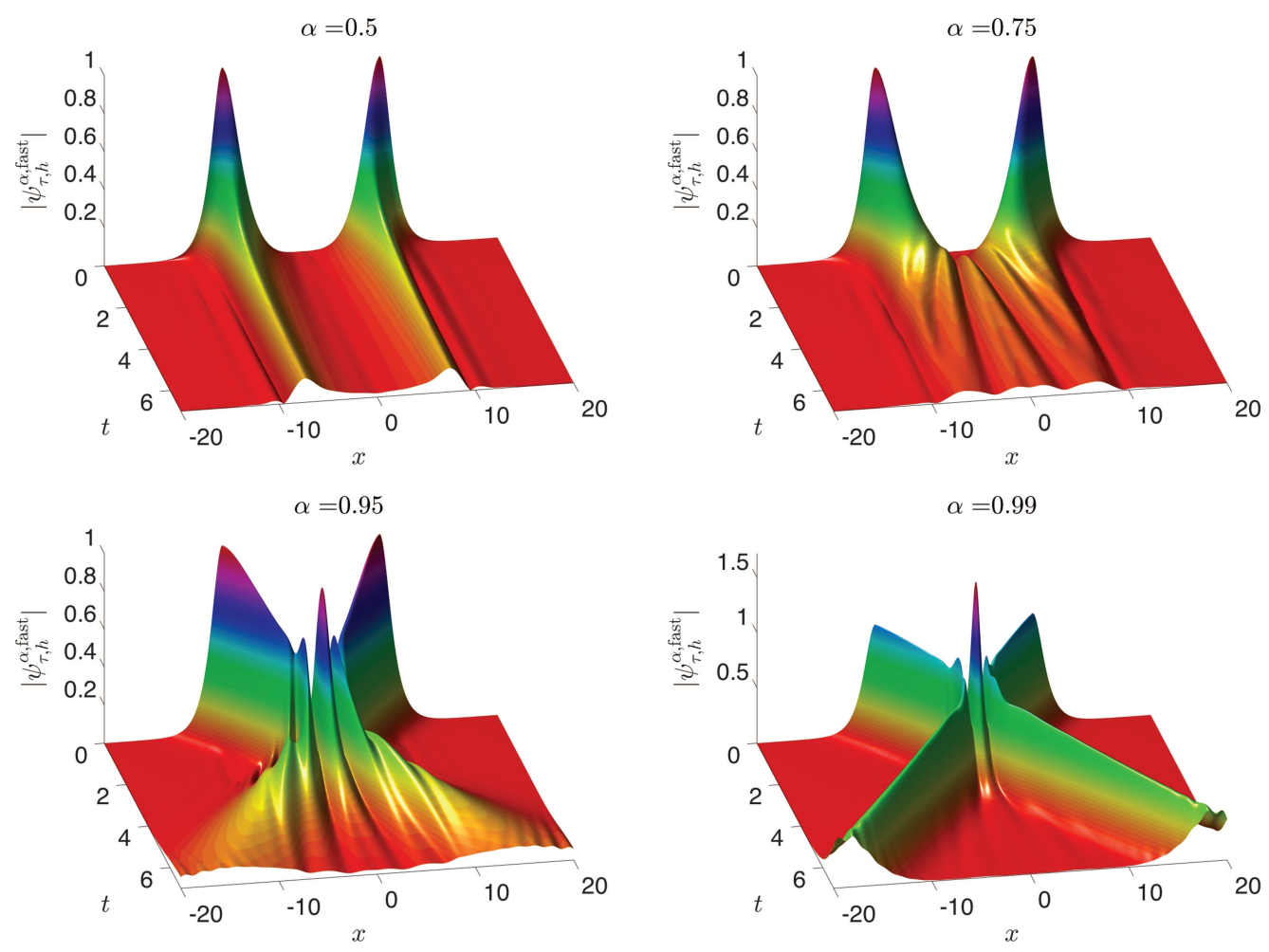

Figure 7: Example 5.3: Evolution of $|\psi|$ for $\alpha=0.5,0.75,0.95$ and 0.99 .

For the standard nonlinear equation with $\alpha=1$, the two soliton waves collide and keep their own shapes moving away after the collision. This can be observed here when $\alpha$ is close to 1 . For smaller values of $\alpha$, this property is loss and the waves do not even cross. At the same time, some waves are created, with high oscillations corresponding to fluctuations and suggesting the appearance of a decoherence phenomena that depends on $\alpha$. 
Example 5.4. In this last example, we consider the TFNSE with a cubic-plus-quintic nonlinearity $f\left(|\psi|^{2}\right) \psi=2|\psi|^{2} \psi+|\psi|^{4} \psi$ and $V(x)=0$. The initial data is built as the superposition of two Gaussian functions

$$
\psi_{0}=\mathbf{e}^{-3(x-2)^{2}}+\mathbf{e}^{-3(x+2)^{2}} .
$$

The interval of computation is ] $-15,15$ [ and the final time is $T=3$. The time step is $\tau=2 \times 10^{-3}$ (for $N=1500$ points) and the spatial mesh size is equal to $h=1.5 \times 10^{-2}$ (with $J=2 \times 10^{3}$ points). For the $\mathrm{ABC}$, we consider $s_{0}=20$.

The dynamics of the solution is plotted on Fig. 8 for various values of $\alpha$. While the two waves clearly mixed for $\alpha$ close to 1 with many oscillations and then largely spread out, they tend to form just one smooth wave after a short time, propagating without being deformed. The support tends to be smaller when $\alpha$ decays.
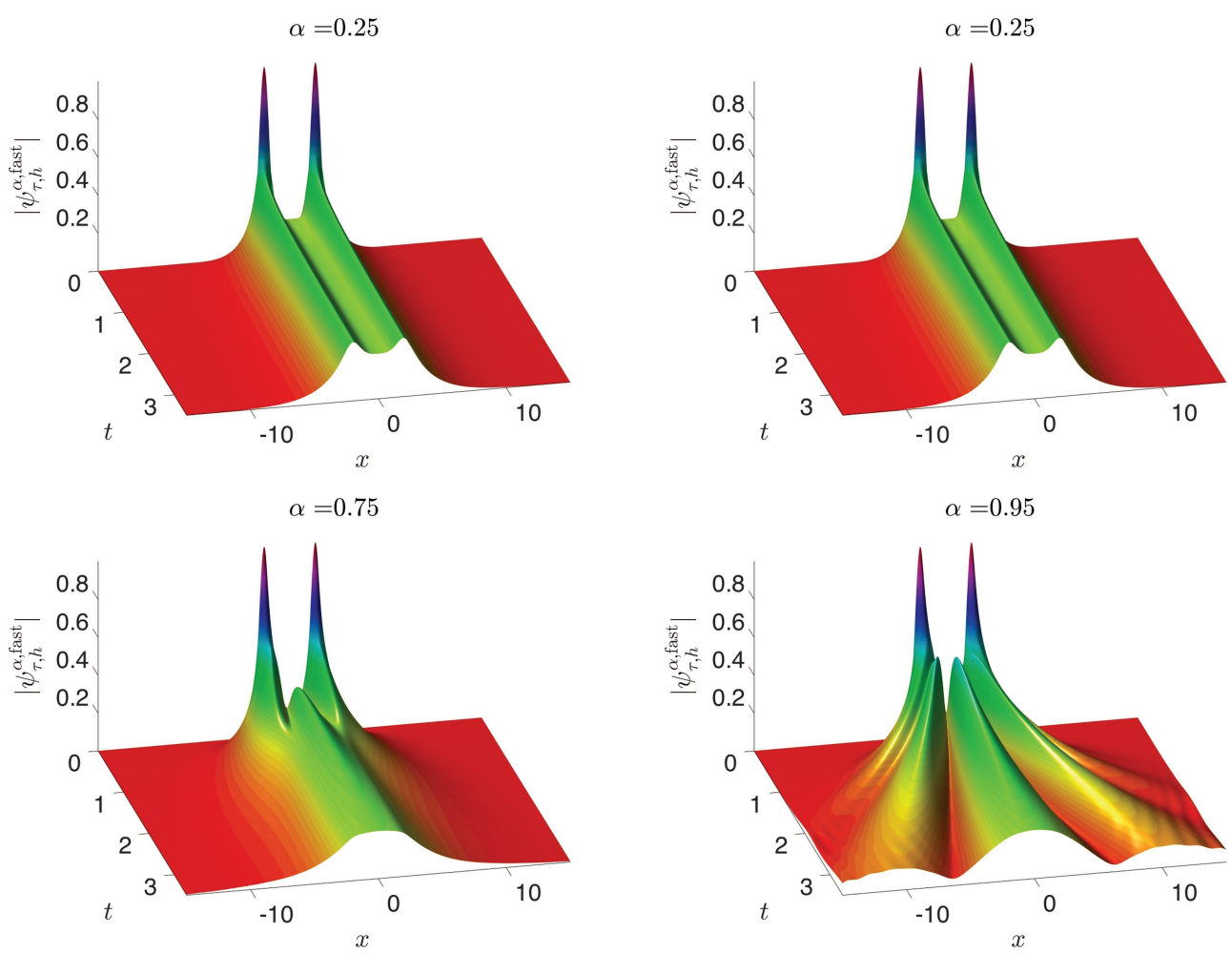

Figure 8: Example 5.4: Evolution of $|\psi|$ for $\alpha=0.25,0.5,0.75$ and 0.95 .

We point out here on the fact that the proposed ABCs are not exact, and therefore lead to existing small reflections at the fictitious boundary while the wave strikes the boundaries (see e.g. Figs. 6, 7 and 8). 


\section{Conclusion}

In this paper, we studied the computation of the time fractional nonlinear Schrödinger equation (TFNSE) on unbounded domain. First, we generalized the construction of approximate nonlinear $\mathrm{ABC}$ s through the unified approach proposed in $[47,48]$ to compute the solution to the TFNSE. In addition, we analyzed the stability of the reduced problem with the approximate nonlinear $\mathrm{ABCs}$ and the convergence of the linearized finite difference scheme by using the discrete fractional-type Gronwall inequality. To speed-up the computations, we used the fast evaluation of the fractional Caputo derivative given in [20]. We presented some numerical examples to verify the performance of the proposed numerical methods. The extension of the method to the two-dimensional TFNSE on unbounded domain and its fast evaluation for high-order numerical schemes will be discussed in a future work.

\section{Acknowledgments}

The research of Jiwei Zhang was supported by the NSFC under grants 11771035, 91430216 and U1530401. Dongfang Li has been supported by the NSFC under grants Nos. 11571128, 11771162. Xavier Antoine received the support of the French ANR grant BOND (ANR-13-BS01-0009-01) and the LIASFMA (funding from the University of Lorraine).

\section{References}

[1] B. Alpert, L. Greengard, and T. Hagstrom, Rapid evaluation of nonreflecting boundary kernels for time-domain wave propagation, SIAM J. Numer. Anal., 37 (2000), 1138-1164.

[2] X. Antoine, A. Arnold, C. Besse, M. Ehrhardt and A. Schädle, A review of transparent and artificial boundary conditions techniques for linear and nonlinear Schrödinger equations, Commun. Comput. Phys., 4 (4) (2008), 729-796.

[3] X. Antoine and C. Besse, Unconditionally stable discretization schemes of non-reflecting boundary conditions for the one-dimensional Schrödinger equation, J. Comput. Phys. 188 (2003), pp. 157-175.

[4] X. Antoine, C. Besse and P. Klein, Absorbing boundary conditions for the one-dimensional Schrödinger equation with an exterior repulsive potential, J. Comput. Phys., 228(2) (2009), 312-335.

[5] X. Antoine, C. Besse and P. Klein, Absorbing boundary conditions for general nonlinear Schrödinger equations, SIAM J. Sci. Comput., 33 (2) (2011),1008-1033.

[6] X. Antoine, Q. Tang and J. Zhang, On the numerical solution and dynamical laws of nonlinear fractional Schrödinger/Gross-Pitaevskii equations, International Journal of Computer Mathematics, 95 (6-7) (2018), 1423-1443.

[7] A.A. Awotunde, R.A. Ghanam, N. Tatar, Artificial boundary condition for a modified fractional diffusion problem, Bound. Value Probl., 1 (2015), 1-17.

[8] G.A. Baker, and P. Graves-Morris, Padé Approximations, 2nd edition, Cambridge University Press, 1996. 
[9] A. Bamberger, B. Engquist, L. Halpern and P. Joly, Higher order paraxial wave equation approximations in heterogeneous media, SIAM J. Appl. Math., 48 (1988), 129-154.

[10] M. Bhatti, Fractional Schrödinger wave equation and fractional uncertainty principle, International J. Contem. Math. Sci., 2 (2007), 943-950.

[11] A.H. Bhrawy and M.A. Abdelkawy, A fully spectral collocation approximation for multidimensional fractional Schrödinger equations, J. Comput. Phys., 294 (2015), 462-483.

[12] H. Brunner, H. Han and D. Yin, Artificial boundary conditions and finite difference approximations for a time-fractional diffusion-wave equation on a two-dimensional unbounded spatial domain, J. Comput. Phys., 276 (2014), 541-562.

[13] W. Cao, Z. Zhang and G. Karniadakis, Time-splitting schemes for fractional differential equations I: smooth solutions, SIAM J. Sci. Comput., 37(4) (2015), A1752-A1776.

[14] J. Dea, Absorbing boundary conditions for the fractional wave equation, Appl. Math. Comput. 219 (2013), 9810-9820.

[15] J. Dong and M. Xu, Space-time fractional Schrödinger equation with time-independent potentials, J. Math. Anal. Appl., 344(2)(2008), 1005-1017.

[16] B. Engquist and A. Majda, Absorbing boundary conditions for the numerical simulation of waves, Math. Comp., 31 (1977), 629-357.

[17] R. Garrappa, I. Moret and M. Popolizio, Solving the time-fractional Schrödinger equation by Krylov projection methods, J. Comput. Phys. 293 (2015), 115-134.

[18] R. Ghaffari and S.M. Hosseini, Obtaining artificial boundary conditions for fractional subdiffusion equation on space two-dimensional unbounded domains. Comput. Math. Appl., 68(1) (2014), 13-26.

[19] B. Hicdurmaz and A. Ashyralyev, A stable numerical method for multidimensional time fractional Schrödinger equation, Comput. Math. Appl., 72 (2016), 1703-1713.

[20] S. Jiang, J. Zhang, Q. Zhang and Z. Zhang, Fast evaluation of the Caputo fractional derivative and its applications to fractional diffusion equations, Commun. Comput. Phys., 21 (2017) 650-678.

[21] W. Jones and W. Thron, Continued Fractions, Analytic Theory and Applications, AddisonWesley Publishing Company, 1980.

[22] R. Ke, M. Ng, and H. Sun, A fast direct method for block triangular Toeplitz-like with tridiagonal block systems from time-fractional partial differential equations, J. Comput. Phys., 303 (2015), 203-211.

[23] N. Khan, M. Jamil and A. Ara, Approximate solutions to time-fractional Schrödinger equation via homotopy analysis method, ISRN Math. Phys., 2012, Article ID 197068.

[24] H. Kreiss and J. Lorenz, Initial boundary value problems and the Navier-Stokes equations, III. Series: Pure and applied mathematics, Academic Press, Vol. 136, 1989.

[25] J. Kuska, Absorbing boundary conditions for the Schrödinger equation on finite intervals, Phys. Rev. B, 46(1992), 5000.

[26] D. Kusnezov, A. Bulgac, and G. Dang, Quantum Lévy processes and fractional kinetics, Phys. Rev. Lett., 82 (1999), 1136.

[27] T. Langlands and B. Henry, The accuracy and stability of an implicit solution method for the fractional diffusion equation, J. Comput. Phys., 205 (2005), 719-736.

[28] N. Laskin, Fractional quantum mechanics, Phys. Rev. E, 62(2000), 3135-3145.

[29] N. Laskin, Fractional Schrödinger equation, Phys. Rev. E, 66 (2002), 056108.

[30] C. Li, W. Deng, and $\mathrm{Y} . \mathrm{Wu}$, Numerical analysis and physical simulations for the time fractional radial diffusion equation, Comput. Math. Appl., 62 (2011), 1024-1037.

[31] D. Li, H. Liao, W. Sun, J. Wang, J. Zhang, Analysis of L1-Galerkin FEMs for time-fractional 
nonlinear parabolic problems, Commun. Comput. Phys., 24 (2018), 86-103.

[32] D. Li, J. Wang, J. Zhang, Unconditionally convergent L1-Galerkin FEMs for nonlinear timefractional Schrödinger equations, SIAM. J. Sci. Comput., 39 (2017), A3067-A3088.

[33] D. Li, J. Zhang and Z. Zhang, The numerical computation of the time fractional Schrödinger equation on unbounded domain, Comput. Methods Appl. Math., DOI: 10.1515/cmam-2017$0038,2017$.

[34] Y. Lin and C. Xu, Finite difference/spectral approximations for time-fractional diffusion equation, J. Comput. Phys., 225 (2007), 1533-1552.

[35] A. Lomin, Fractional-time quantum dynamics, Phys. Rev. E, 62 (2000), 3135-3145.

[36] X. Lu, H. Sun, and H. Pang, Fast approximate inversion of a block triangular Toeplitz matrix with applications to fractional subdiffusion equations, J. Numer. Lin. Alg. Appl., 22 (2015), 866-882.

[37] Y.Y. Lu, A Padé approximation method for square roots of symmetric positive definite matrices, SIAM J. Math. Anal. Appl., 19(3)(1998), 833-845.

[38] C. Lubich and A. Schädle, Fast convolution for nonreflecting boundary conditions, SIAM J. Sci. Comput. 24 (2002) 161-182.

[39] M. Naber, Time fractional Schrödinger equation, J. Math. Phys., 45 (2004), 3339-3352.

[40] B. Narahari Achar, Bradley T. Yale, and John W. Hanneken, Time fractional Schrödinger equation revisited, Adv. Math. Phys., (2013) Article ID 290216.

[41] Z. Odibat, S. Momani, and A. Alawneh, Analytic study on time-fractional Schrödinger equations: exact solutions by GDTM, J. Phys.: Conference Series 96 (2008), 012066.

[42] I. Podlubny, Fractional Differential Equations, Academic Press, San Diego, 1999.

[43] H. Spohn, Kinetic equations from Hamiltonian dynamics: Markovian limits, Rev. Mod. Phys. 52(1980), 569-615.

[44] Z. Sun and X. Wu, The stability and convergence of a difference scheme for the Schrödinger equation on an infinite domain by using artificial boundary conditions, J. Comput. Phys., 214 (2006), 209-223.

[45] S. Wang and M. Xu, Generalized fractional Schrödinger equation with space-time fractional derivatives, J. Math. Phys., 48(4) (2007), 043502.

[46] B. West, M. Bologna, and P. Grigolini, Physics of Fractal Operators, Springer, New York, 2002.

[47] J. Zhang, Z. Xu, X. Wu, Unified approach to split absorbing boundary conditions for nonlinear Schrödinger equations, Phys. Rev. E, 78 (2008), 026709.

[48] J. Zhang, Z. Xu, X. Wu, Unified approach to split absorbing boundary conditions for nonlinear Schrödinger equations: Two dimensional case, Phys. Rev. E, 79 (2009), 046711.

[49] I. Podlubny, Fractional Differential Equations, Academic Press, San Diego, 1999.

[50] J. Zhang, Z. Xu, X. Wu and D. Wang, Stability analysis of local absorbing boundary conditions for general nonlinear Schrödinger equations in one and two dimensions, J. Comput. Math., 35 (2017) 1-18.

[51] C. Zheng, Approximation, stability and fast evaluation of exact artificial boundary condition for one-dimensional heat equation, J. Comput. Math., 25 (2007), 730-745. 